Development of Models and Software for Liquidus Temperatures RECEIVED of Glasses of HWVP Products:
P. R. Hrma
P. Wu
J. D. Vienna
G. Eriksson

A. D. Pelton

S. Degtiarev

March 1996

Prepared for the U.S. Department of Energy under Contract DE-AC06-76RLO 1830

Pacific Northwest National Laboratory Operated for the U.S. Department of Energy by Battelle Memorial Institute

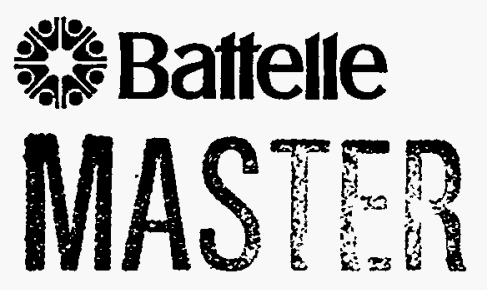




\title{
Development of Models and Software for Liquidus Temperatures of Glasses of HWVP Products: Final Report
}

\author{
P. R. Hrma \\ J. D. Vienna \\ A. D. Pelton* \\ P. $\mathrm{Wu}^{*}$ \\ G. Eriksson* \\ S. Degtiarev*
}

March 1996

Prepared for

the U.S. Department of Energy

under Contract DE-AC06-76RLO 1830

Pacific Northwest National Laboratory

Richland, Washington 99352

*Centre for Research in Computational Thermochemistry, Ecole Polytechnique

Montreal, Quebec, Canada

Reprint of historical document PVTD-C95-02.010, daled September 1995. Prepared for PNL by the Centre for Research in Computational Thermochemistry, Ecole Polytechnique, Montreal, Quebec, Canada. Data, formatting, and ocher conventions reflect standards, at the original date of printing. Technical peer reviews and editorial reviews may not have been performed. 


\title{
DISCLAISAER
}

This report wes prepared as an account of work sponsored by an agency of the United Staies Government. Neither the United Staies Covernment nor any agency thereof, nor Eażelle Memorial Instituie, nor any of their emplojees, makes any . warranty, express or implied, or assumes any legal liability or responsibility for the accuracy, completeness, or.usefulness of any information, apparatus, product, or process disclosed, or represents that its use would not iniringe privately owned rights. Reference herein io any speciñc commercial product, process, or service by irade name, itacemark, menufacturer, or chitenvise dües not necessarily constitute or imply its endorsement, recommendation, or favoring by the United States Government or any agency thereof, or Banelle Memorial instiute. The views and opinions of authors expressed herein do not necessarily state or reflect those of the United States Government or any agency thereof.

\author{
PACIFIC NORTHWEST NATIONAL LABORATORY \\ operaied by \\ BATTELLE \\ for the \\ UNITED STATES DEPARTMENT OF ENERGY \\ under Contract DE-ACO6-76RLO 1830
}

\section{Printed in the United Stales of America}

Arailable to DOE and DOE contractors from the

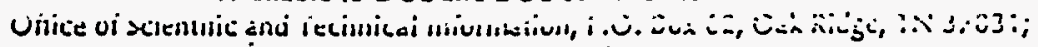
prices, arailable from (615) 576-6401.

Available to the public from the National Technical Information Service, U.S. Department of Commerce, 5285 Port Royal Rd., Springfield, VA 22161 


\section{TABLE OF CONTENTS}

1 INTRODUCTION/SUMMARY

2 OPTIMIZATION OF BASE GLASS ...................... . . 7

$2.1 \mathrm{Na}_{2} \mathrm{O}-\mathrm{B}_{2} \mathrm{O}_{3}-\mathrm{SiO}_{2}$ system $\ldots \ldots \ldots \ldots \ldots \ldots \ldots \ldots \ldots \ldots \ldots \ldots$

$2.2 \mathrm{Na}_{2} \mathrm{O}-\mathrm{Al}_{2} \mathrm{O}_{3}-\mathrm{SiO}_{2}$ system $\ldots \ldots \ldots \ldots \ldots \ldots \ldots \ldots \ldots \ldots \ldots \ldots$

$2.3 \mathrm{Na}_{2} \mathrm{O}-\mathrm{Fe}_{2} \mathrm{O}_{3}-\mathrm{SiO}_{2}$ system $\ldots \ldots \ldots \ldots \ldots \ldots \ldots \ldots \ldots \ldots \ldots \ldots$

2.4 Other Systems with $\mathrm{Al}_{2} \mathrm{O}_{3}$ and $\mathrm{Fe}_{2} \mathrm{O}_{3} \ldots \ldots \ldots \ldots \ldots \ldots \ldots \ldots \ldots$

$2.5 \mathrm{Na}_{2} \mathrm{O}-\mathrm{ZrO}_{2}-\mathrm{SiO}_{2}$ system land compound $\left.\mathrm{Na}_{2} \mathrm{ZrSi}_{2} \mathrm{O}_{7}\right) \ldots \ldots \ldots \ldots \ldots$

2.6 $\mathrm{Na}_{2} \mathrm{O}-\mathrm{CaO}-\mathrm{SiO}_{2}$ system (and solid solution $\mathrm{Na}_{2} \mathrm{Ca}_{2} \mathrm{Si}_{3} \mathrm{O}_{9}-\mathrm{Na}_{4} \mathrm{CaSi}_{3} \mathrm{O}_{9}$ ) $\quad \ldots \quad 11$

2.7 Clinopyroxene $\left(\mathrm{NaFeSi}_{2} \mathrm{O}_{0} \cdot \mathrm{CaMgSi}_{2} \mathrm{O}_{8}\right.$ ) solid solution $\ldots \ldots \ldots \ldots \ldots$

2.8. Optimization of CVS liquidus calculations ............... 12

3 INCLUDING $\mathrm{Fe}^{2+}$ IN THE DATABASES .................. 12

4 INCLUDING CHROME IN THE DATABASES ................ 13

$5 \quad$ INCLUDING NICKEL IN THE DATABASES $\ldots \ldots \ldots \ldots \ldots \ldots \ldots \ldots$

6 INCLUDING MANGANESE IN THE DATABASES ............... 13

7 . CALCULATIONS FOR CVS GLASSES .................. 14

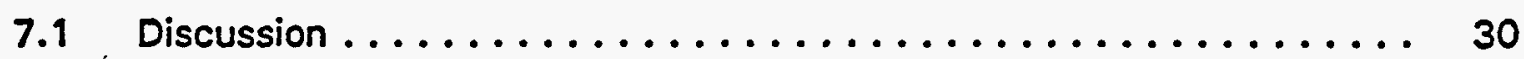

8 THE SPINEL PHASE $\ldots \ldots \ldots \ldots \ldots \ldots \ldots \ldots \ldots \ldots \ldots \ldots \ldots \ldots \ldots \ldots$

9 SOLUBILITY OF $\mathrm{SO}_{3}, \mathrm{PO}_{4}, \mathrm{Cl}, \mathrm{F}, \mathrm{I}$ AND $\mathrm{H}_{2} \mathrm{O}$ IN GLASSES . . . . . . . . . 32

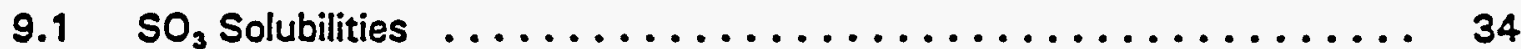

9.2 Phosphate solubilities ....................... 41

9.3 Halide solubilities ........................... 44

$9.4 \mathrm{H}_{2} \mathrm{O}$ Solubilities $\ldots \ldots \ldots \ldots \ldots \ldots \ldots \ldots \ldots \ldots \ldots \ldots \ldots \ldots$

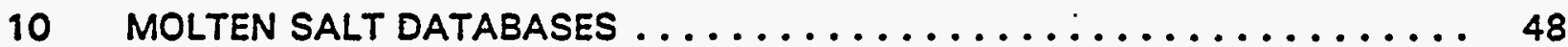

11 REFERENCES .............................. 49 
In an earlier report [92 Pel] was described the development of software and thermodynamic databases for the calculation of liquidus temperatures of glasses of HWVP products containing the components $\mathrm{SiO}_{2}-\mathrm{B}_{2} \mathrm{O}_{3}-\mathrm{Na}_{2} \mathrm{O}-\mathrm{Li} 2 \mathrm{O}-\mathrm{CaO}-\mathrm{MgO}-\mathrm{Fe}_{2} \mathrm{O}_{3}-\mathrm{Al}_{2} \mathrm{O}_{3}-\mathrm{ZrO}_{2}$ "others".

The software package developed at that time consisted of the EQUILIB program of the F*A $C$ " $T$ computer system with special input/output routines. Since then, Battelle has purchased the entire $F^{*} A{ }^{*} C^{*} T$ computer system, and this fully replaces the earlier package. Furthermore, with the entire $F$ " $A$ " $C$ *T system, additional calculations can be performed such as calculations at fixed $\mathrm{O}_{2}, \mathrm{SO}_{2}$, etc. pressures, or graphing of output. Furthermore, the public $F * A$ * $C$ "T database of over 5000 gaseous species and condensed phases is now accessible.

The private databases for the glass and crystalline phases were developed for Battelle by optimization of thermodynamic and phase diagram data. That is, all available data for 2and 3-component sub-systems of the 9-component oxide system were collected, and parameters of model equations for the thermodynamic properties were found which best reproduce all the data. For representing the thermodynamic properties of the glass as a function of composition and temperature, the modified quasichemical model was used. This model was described in the earlier report [92 Pel] along with all the optimizations. With the model, it was possible to predict the thermodynamic properties of the 9-component glass, and thereby to calculate liquidus temperatures. Liquidus temperatures measured by Battelle for 123 CVS glass compositions were used to test the model and to refine the model by the addition of further parameters.

In the present report, we describe the continuation of this work.

First, the optimization of the base 9-component glass was extensively re-examined and improved as will be discussed in Section 2. Many binary and ternary systems were completely re-optimized, and our treatment of $\mathrm{Al}_{2} \mathrm{O}_{3}$ and $\mathrm{Fe}_{2} \mathrm{O}_{3}$ in the model was revised to better account for the charge compensation effect. The clinopyroxene phase has now been modeled as a solid solution $\mathrm{NaFeSi}_{2} \mathrm{O}_{6}-\mathrm{CaMgSi} \mathrm{O}_{6}$ and new thermodynamic data for acmite have been used. A sodium-calcium silicate solid solution has also been included, as has the compound $\mathrm{Na}_{2} \mathrm{ZrSi}_{2} \mathrm{O}_{7}$.

Chrome, nickel, manganese and $\mathrm{Fe}^{2+}$ were added to the database through optimization of a large amount of data as described below in Section 3-6. A model for the corundum solid solution $\left(\mathrm{Cr}_{2} \mathrm{O}_{3}-\mathrm{Fe}_{2} \mathrm{O}_{3}-\mathrm{Al}_{2} \mathrm{O}_{3}\right)$ was also developed. Data for $\mathrm{Cr}, \mathrm{Ni}$ and $\mathrm{Fe}$ in other solid compounds and solutions were also optimized, but these phases were never calculated to form as primary phases over the range of composition of the CVS glasses and so they were not included in the databases prepared for Battelle.

A model was also developed for the ( $\mathrm{Fe}, \mathrm{Ni}, \mathrm{Mn}$ ) $(\mathrm{Fe}, \mathrm{Al}, \mathrm{Cr}, \mathrm{Mn}\rangle_{2} \mathrm{O}_{4}$ spinel phase, and parameters were obtained by optimization as discussed in Section 8. 
Calculated liquidus temperatures and primary phases are compared with the experimental results in Section 7. Agreement is significantly improved over that in the earlier report. The comparison of calculations and experimental results is discussed in detail in Section 7.1. Comparisons of calculated and experimental spinel liquidus temperatures are discussed in Section 8.

Bi was not added to the database as originally proposed because, following discussions with P. Hrma, it was decided to include $\mathrm{Mn}$ in the spinel model instead.

A model for sulfate, phosphate, chloride, fluoride, iodide and water in dilute solutions $(<10 \mathrm{wt.} \%)$ in glass was developed. This required re-programming of a number of $F * A$ * $C * T$ software modules.

Calculations of the solubilities of these constituents are compared to available data in Section 9. Agreement is generally very good, and in most cases this agreement is achieved with no adjustable parameters. Solubilities in equilibrium with a gas phase (containing $\mathrm{SO}_{2}$, $\mathrm{O}_{2} \mathrm{H}_{2} \mathrm{O}$, etc.) can be calculated with the ACT option of the EQUILIB program. Solubilities in equilibrium with solid or liquid sulphates, phosphates and halides can also be calculated. In the case of phosphates, optimized data for the solid phosphates are included in Battelle's private one-component database. For liquid sulphates and halides, which can form molten salt solutions, use either the /SOLN-SALT/ solution from the public F*A*C "T database, or the Li, $\mathrm{Na}, \mathrm{Mg}, \mathrm{Ca} / \mathrm{F}, \mathrm{SO}_{4}$ molten salt solution which has been prepared by optimization and is included in the private SOLUTION database. Data for pure solid and liquid sulfates and halides can be taken from the public $F * A * C * T$ database.

The optimization of the $\mathrm{Li}, \mathrm{Na}, \mathrm{Mg}, \mathrm{Ca} / \mathrm{F}, \mathrm{SO}_{4}$ molten salt phase is discussed in Section 10.

A list of the solutions in the optimized private SOLUTION database which has been prepared is given in Table 1, while a list of the contents of the private one-component databases prepared for Battelle is given in Table 2. In the species selection step of the EQUILIB program one should select only these solutions and compounds as well as the /SOLNSALT/ phase and, if required, data for pure liquid or solid halides and sulfates and any gases from the public $F * A$ * $C * T$ databases. No other species or solutions from the public $F * A * C * T$ databases should be required, and their selection could cause problems of compatibility of data. Of course, a saving in computation time can be realized if species which are certain not to form in a given case are not selected. Note also that after an EQUILIB calculation, if one enters "LIST", then the calculated activities of all species and solution components are displayed. 


\section{Table 1}

Solutions in Private Battelle Database

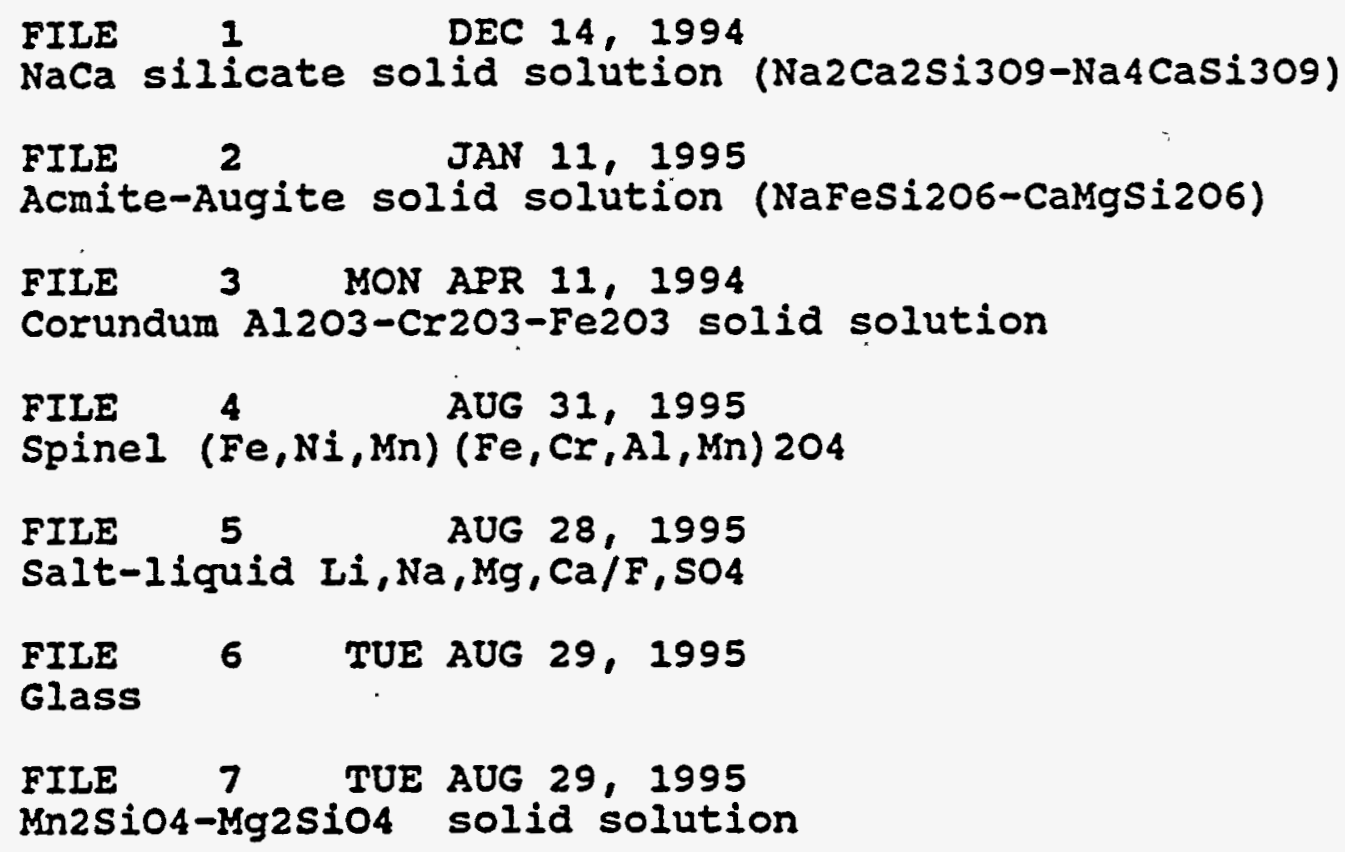


Table 2

Compounds in Private Battelle Database

Species found in USERBASE

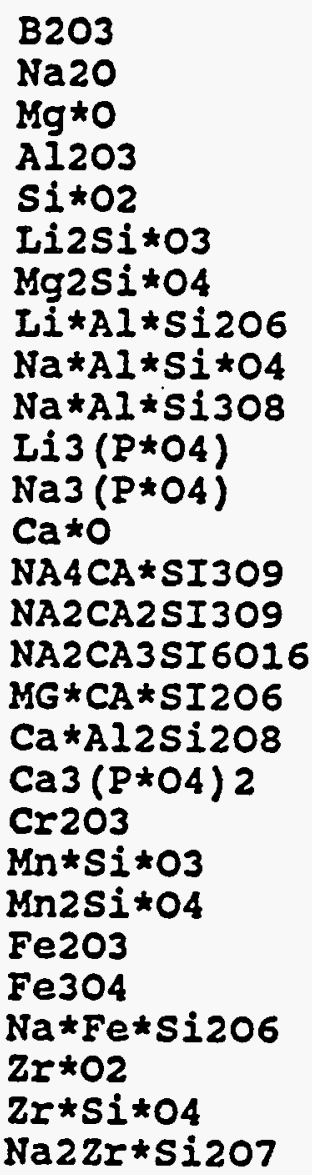

: SI II

: S1 S2 S3 I1

: S1 II GI

$\begin{array}{lllll}51 & 52 & 53 & 54 & \text { L1 }\end{array}$

$\begin{array}{lllllll}51 & 52 & 53 & S 4 & S 5 & S 6 & \text { L1 }\end{array}$

Li2Si*03

S1

S1

S1

S1

S1 52

51

: 51

S1

: 51

: 51

: $\$ 1$

: 51

: 51

: S1 S2

: S1 LI

: SI

: S1 II

: 51 S2

: 51 S2 II

: 51

: S1

: 51

$\mathrm{Na2Zr*Si207}$

S2 S3 II G1

: S1 


\section{OPTIMIZATION OF BASE GLASS}

The optimization of the base glass $\left(\mathrm{SiO}_{2}-\mathrm{B}_{2} \mathrm{O}_{3}-\mathrm{Na}_{2} \mathrm{O}-\mathrm{Li} \mathrm{i}_{2} \mathrm{O}-\mathrm{CaO}-\mathrm{MgO}-\mathrm{Fe}_{2} \mathrm{O}_{3}-\mathrm{Al}_{2} \mathrm{O}_{3}-\mathrm{ZrO}_{2}\right)$ was discussed in the earlier report $[92$ Pel]. Since then, the optimization has been extensively re-examined and improved.

A number of ternary sub-systems have been re-optimized with attention paid only to. those composition regions where the basicity falls within the range of basicities of Battelle's CVS glasses because, from the equations of the model, only optimizations within these composition regions will have any influence on the multicomponent calculations. According to the model, "basicity" is defined as:

$\left(n_{\mathrm{SO}_{2}}+2 n_{\mathrm{B}_{2} \mathrm{O}_{3}}+n_{\mathrm{ZO}_{2}}\right) /\left(n_{\mathrm{SO}_{2}}+2 n_{\mathrm{B}_{2} \mathrm{O}_{3}}+n_{\mathrm{ZO}_{2} \mathrm{O}_{2}}+2 n_{\mathrm{Na}_{2} \mathrm{O}}+2 n_{\mathrm{L}_{2} \mathrm{O}}+n_{\mathrm{COO} \mathrm{O}}+n_{\mathrm{MgO}_{9} \mathrm{O}}+2 n_{\mathrm{Al}_{2} \mathrm{O}_{3}}+2 n_{\mathrm{FO}_{2} \mathrm{O}_{3}}\right)$

\section{$2.1 \quad \mathrm{Na}_{2} \mathrm{O}-\mathrm{B}_{2} \mathrm{O}_{3}-\mathrm{SiO}_{2}$ system}

In the case of the $\mathrm{Na}_{2} \mathrm{O}-\mathrm{B}_{2} \mathrm{O}_{3}-\mathrm{SiO}_{2}$ system, the following optimized parameters were obtained. Calculated and experimental [51 Mor] liquidus points are compared in Table 3.

Since the optimization only applies for the range of basicity of the CVS glasses, the miscibility gap at low $\mathrm{Na}_{2} \mathrm{O}$ contents in the $\mathrm{Na}_{2} \mathrm{O}-\mathrm{B}_{2} \mathrm{O}_{3}-\mathrm{SiO}_{2}$ system is not correctly reproduced.

$$
\begin{aligned}
& \omega_{\text {NaSi (B) }}^{\text {bi }}=-83.68 \mathrm{~kJ} / \mathrm{mol} \\
& \omega_{\text {SNa (B) }}^{11}=-125.52 \mathrm{~kJ} / \mathrm{mol} \\
& \omega_{\text {NoB (S) }}^{\text {ol }}=75.31 \mathrm{~kJ} / \mathrm{mol}
\end{aligned}
$$




\section{Table 3}

Calculated and Experimental 151 Morl Liquidus Points in the $\mathrm{Na}_{2} \mathrm{O}-\mathrm{B}_{2} \mathrm{O}_{3}-\mathrm{SiO}_{2}$ System

\begin{tabular}{|c|c|c|c|c|c||}
\hline \multirow{2}{*}{$\begin{array}{c}\text { Point Number } \\
\text { (from (51 Mor]) }\end{array}$} & \multicolumn{3}{|c|}{ Composition (wt.\%) } & \multicolumn{2}{c|}{$T^{\circ} \mathrm{C}$ ) } \\
\cline { 2 - 6 } & $\mathrm{Na}_{2} \mathrm{O}$ & $\mathrm{B}_{2} \mathrm{O}_{3}$ & $\mathrm{SiO}_{2}$ & expt. & calc. \\
\hline 102 & 30.8 & 15.8 & 53.4 & 706 & 680 \\
104 & 28.3 & 11.4 & 60.3 & 710 & 687 \\
105 & 30.0 & 10.0 & 60.0 & 755 & 722 \\
106 & 34.0 & 6.0 & 60.0 & 805 & 797 \\
& & & & & \\
107 & 49.2 & 21.2 & 29.6 & 854 & 869 \\
108 & 49.4 & 15.2 & 35.4 & 916 & 949 \\
109 & 49.7 & 10.3 & 40.0 & 966 & 1002 \\
110 & 35.9 & 15.4 & 4.8 .7 & 769 & 712 \\
111 & 42.0 & 18.2 & 39.8 & 784 & 779 \\
& & & & & \\
87 & 16.3 & 30.5 & 47.2. & 787 & 835 \\
89 & 22.0 & 28.0 & 50.0 & 760 & 736 \\
90 & 19.0 & 26.3 & 54.7 & 771 & 812 \\
92 & 21.0 & 23.6 & 55.4 & 784 & 778 \\
93 & 26.5 & 12.9 & 60.6 & 676 & 671 \\
\hline
\end{tabular}




\section{$2.2 \mathrm{Na}_{2} \mathrm{O} \cdot \mathrm{Al}_{2} \mathrm{O}_{3}-\mathrm{SiO}_{2}$ system}

In the $\mathrm{Na}_{2} \mathrm{O} \cdot \mathrm{Al}_{2} \mathrm{O}_{3}-\mathrm{SiO}_{2}$ system, a "charge compensation effect" is operative, whereby an $\mathrm{Al}$ atom can replace Si on a network site only if paired with a $\mathrm{Na}$ ion. This means that the structure and properties of the glasses are very different on either side of a line where the $\mathrm{Na} / \mathrm{Al}$ molar ratio is 1.0. Since this ratio is greater than 1.0 for nearly all CVS glasses, it was decided to optimize only this region. This was found to be much easier if the quasichemical "charge parameter" of alumina was set equal to that of sodium (0.3444). Furthermore, since compositions on the $\mathrm{Al}_{2} \mathrm{O}_{3}-\mathrm{SiO}_{2}$ and $\mathrm{Na}_{2} \mathrm{O}-\mathrm{Al}_{2} \mathrm{O}_{3}$ systems are not in the ranges of composition - (either of $\mathrm{Na} / \mathrm{Al}$ ratio or of basicity) of interest, the binary parameters of these systems were chosen only with a view to optimizing the ternary system. The following optimized parameters were obtained

$$
\begin{aligned}
& \omega_{\text {N2Al }}=-449.78 \mathrm{~kJ} / \mathrm{mol} \\
& \omega_{\text {Alsi }}=-190.255 y_{s i}^{7} \mathrm{~kJ} / \mathrm{mol} \\
& \omega_{\mathrm{N} \omega \mathrm{J}}^{\text {of }(S)}=-167.36 \mathrm{~kJ} / \mathrm{mol}
\end{aligned}
$$

A comparison of calculated and experimental $600 \mathrm{Osb}$ ] invariant points is given in Table 4.

A satisfactory optimization of the nepheline $(\mathrm{NaAlSiO})$ liquidus of the $\mathrm{Na}_{2} \mathrm{O}-\mathrm{Al}_{2} \mathrm{O}_{3}-\mathrm{SiO}_{2}$ system was not obtained. Consequently, liquidus temperatures of CVS glasses with compositions for which nepheline is a possible primary phase are not well calculated as will be discussed in Section 7.

\section{$2.3 \quad \mathrm{Na}_{2} \mathrm{O}-\mathrm{Fe}_{2} \mathrm{O}_{3}-\mathrm{SiO}_{2}$ system}

In the $\mathrm{Na}_{2} \mathrm{O}-\mathrm{Fe}_{2} \mathrm{O}_{3}-\mathrm{SiO}_{2}$ system, a charge compensation effect is also probably operative, although to a leaser degree than in $\mathrm{Na}_{2} \mathrm{O}-\mathrm{Al}_{2} \mathrm{O}_{3}-\mathrm{SiO} \mathrm{O}_{2}$. In any case, the optimization of this system was improved over that reported in the earlier report by setting the "charge parameter" of $\mathrm{FeO}_{3 / 2}$ equal to that of $\mathrm{Na}_{2} \mathrm{O}(0.3444)$. Furthermore, most emphasis was placed on fitting the phase diagram [30 Bow] at low $\mathrm{Fe}_{2} \mathrm{O}_{3}$ contents. More recent data [90 Hol] for the thermodynamic properties of pure acmite $\left(\mathrm{NaFeSi}_{2} \mathrm{O}_{6}\right)$ were employed than were used in the earlier report. $\Delta \mathrm{H}_{298}^{\circ}$ of acmite was later adjusted by $-16.75 \mathrm{~kJ} / \mathrm{mol}$ in the optimization which is within the experimental error limits. One optimized ternary parameter was required:

$$
\omega_{\text {NeSi (Fo) }}^{09}=-50.21 \mathrm{~kJ} / \mathrm{mol}
$$




\section{Table 4}

Comparison of Calculated and Experimental $160 \mathrm{Osb}$ Invariant Points in the $\mathrm{Na}_{2}{ }_{2}-\mathrm{Al}_{2} \mathrm{O}_{3}-\mathrm{SiO}_{2}$ System

\begin{tabular}{|c|c|c|c|c|}
\hline \multicolumn{3}{|c|}{ Composition (wt.\%) } & $\mathrm{T}\left({ }^{\circ} \mathrm{C}\right)$ & Reaction \\
\hline $\mathrm{Na}_{2} \mathrm{O}$ & $\mathrm{Al}_{2} \mathrm{O}_{3}$ & $\mathrm{SiO}_{2}$ & & \\
\hline $\begin{array}{l}\text { expt. } \\
\text { calc. }\end{array}$ & & & $\begin{array}{l}1062 \\
1073\end{array}$ & $L \rightarrow \mathrm{SiO}_{2}+\mathrm{NaAlSi}_{3} \mathrm{O}_{3}$ \\
\hline $\begin{array}{l}\text { expt. } \\
\text { calc. }\end{array}$ & & & $\begin{array}{l}740 \\
750\end{array}$ & $\mathrm{~L} \rightarrow \mathrm{Na}_{2} \mathrm{Si}_{2} \mathrm{O}_{5}+\mathrm{SiO}_{2}+\mathrm{NaAlSi}_{3} \mathrm{O}_{8}$ \\
\hline $\begin{array}{ll}\text { expt. } & 26.0 \\
\text { calc. } & 27.8\end{array}$ & $\begin{array}{l}12.5 \\
11.2\end{array}$ & $\begin{array}{l}61.5 \\
61.0\end{array}$ & $\begin{array}{l}732 \\
735\end{array}$ & $\mathrm{~L} \rightarrow \mathrm{Na}_{2} \mathrm{Si}_{2} \mathrm{O}_{5}+\mathrm{NaAlSi}_{3} \mathrm{O}_{8}+\mathrm{NaAlSiO}_{4}$ \\
\hline $\begin{array}{ll}\text { expt. } & 32.0 \\
\text { calc. } & 31.5\end{array}$ & $\begin{array}{l}10.1 \\
10.4\end{array}$ & $\begin{array}{l}57.9 \\
58.1\end{array}$ & $\begin{array}{l}760 \\
742\end{array}$ & $\mathrm{~L} \rightarrow \mathrm{Na}_{2} \mathrm{Si}_{2} \mathrm{O}_{5}+\mathrm{Na}_{2} \mathrm{SiO}_{3}+\mathrm{NaAlSiO}_{4}$ \\
\hline $\begin{array}{l}\text { expt. } \\
\text { calc. }\end{array}$ & & & $\begin{array}{l}1118 \\
1118\end{array}$ & $L \rightarrow \mathrm{NaAlSi}_{3} \mathrm{O}_{8}$ \\
\hline
\end{tabular}




\subsection{Other Systems with $\mathrm{Al}_{2} \mathrm{O}_{3}$ and $\mathrm{Fe}_{2} \mathrm{O}_{3}$}

The treatment of the components $\mathrm{Al}_{2} \mathrm{O}_{3}$ and $\mathrm{Fe}_{2} \mathrm{O}_{3}$ is now different, since their assumed "charge parameters" in the quasichemical model were changed in order to give better optimizations of the important $\mathrm{Na}_{2} \mathrm{O}-\mathrm{Al}_{2} \mathrm{O}_{3}-\mathrm{SiO}_{2}$ and $\mathrm{Na}_{2} \mathrm{O}-\mathrm{Fe}_{2} \mathrm{O}_{3}-\mathrm{SiO} \mathrm{O}_{2}$ systems. (These systems are important because $\mathrm{Na}_{2} \mathrm{O}$ and $\mathrm{SiO}_{2}$ are both major $\mathrm{CVS}$ glass components.) Because of this, it was necessary to re-optimize all systems containing $\mathrm{Al}_{2} \mathrm{O}_{3}$ or $\mathrm{Fe}_{2} \mathrm{O}_{3}$. The systems which were re-optimized are: $\mathrm{CaO}-\mathrm{Fe}_{2} \mathrm{O}_{3}, \mathrm{Al}_{2} \mathrm{O}_{3}-\mathrm{B}_{2} \mathrm{O}_{3}, \mathrm{Al}_{2} \mathrm{O}_{3}-\mathrm{CaO}, \mathrm{Al}_{2} \mathrm{O}_{3}-\mathrm{MgO}_{2} \mathrm{Al}_{2} \mathrm{O}_{3}-$ $\mathrm{ZrO}_{2}, \quad \mathrm{Na}_{2} \mathrm{O}-\mathrm{Fe}_{2} \mathrm{O}_{3}, \quad \mathrm{SiO}_{2}-\mathrm{Fe}_{2} \mathrm{O}_{3}, \quad \mathrm{Al}_{2} \mathrm{O}_{3}-\mathrm{B}_{2} \mathrm{O}_{3}-\mathrm{SiO}_{2}, \quad \mathrm{Al}_{2} \mathrm{O}_{3}-\mathrm{Li}_{2} \mathrm{O}-\mathrm{SiO}_{2}, \quad \mathrm{CaO}-\mathrm{Al}_{2} \mathrm{O}_{3}-\mathrm{SiO}_{2}$, $\mathrm{MgO}-\mathrm{Al}_{2} \mathrm{O}_{3}-\mathrm{SiO}_{2}$.

\section{$2.5 \mathrm{Na}_{2} \mathrm{O}-\mathrm{ZrO}_{2}-\mathrm{SiO}_{2}$ system land compound $\mathrm{Na}_{2} \underline{\mathrm{ZrSi}}_{2} \mathrm{O}_{7} 1$}

Very few data are available for this system, but a value of $\omega_{\text {NaSi }}^{21}\left(D_{1}\right)=-209.2 \mathrm{~kJ} / \mathrm{mol}$ (- $50 \mathrm{kcal} / \mathrm{mol}$ ) was estimated. A compound $\mathrm{Na}_{2} \mathrm{ZrSi}_{2} \mathrm{O}_{7}$ has been observed which was not included in our earlier report 192 Pell. No thermodynamic data are available for this compound. Accordingly, $\mathrm{Cp}$ was estimated from $\mathrm{Cp}$ of $\mathrm{ZrO}_{2}$ and $\mathrm{ZrSiO}_{4}$, while $\Delta \mathrm{H}_{298}^{\circ}$ and $\mathrm{S}_{298}^{\circ}$ were chosen to best reproduce the observed liquidus temperatures of the CVS glasses.

\section{6 $\mathrm{Na}_{2} \mathrm{O}-\mathrm{CaO}-\mathrm{SiO}_{2}$ system land solid solution $\left.\mathrm{Na}_{2} \mathrm{Ca}_{2} \mathrm{Si}_{3} \mathrm{O}_{0}-\mathrm{Na}_{1} \mathrm{CaSi}_{3} \mathrm{O}_{2}\right)$}

The earlier optimization of this system has been improved. In particular, the assumption of an ideal solid solution between $\mathrm{Na}_{2} \mathrm{Ca}_{2} \mathrm{Si}_{3} \mathrm{O}_{9}$ and $\mathrm{Na}_{4} \mathrm{CaSi}_{3} \mathrm{O}_{2}$ markedly improves the agreement between the calculations and the published phase diagram.

\subsection{Clinopyroxene $1 \mathrm{NaFeSi}_{2} \mathrm{O}_{6}-\mathrm{CaMgSi}{ }_{2} \mathrm{O}_{6}$ L solid solution}

A major improvement in reproducing the observed liquidus temperatures of the CVS glasses was effected by treating the clinopyroxene phase as a solid solution of $1 / 2\left(\mathrm{NaFeSi}_{2} \mathrm{O}_{6}\right)$ and $1 / 2\left(\mathrm{CaMgSi}_{2} \mathrm{O}_{8}\right)$. Thermodynamic data for pure $\mathrm{CaMgSi}_{2} \mathrm{O}_{8}$ were taken from the $F * A * C$ *T database, and a Henrian solution parameter

$$
R T \ln Y_{1 / 2\left(C_{1} H_{0} S_{i} O_{0}\right)}=-26342+19.246 \mathrm{~T} \mathrm{~J} / \mathrm{mol}
$$

was chosen to best reproduce the observed CVS glass liquidus points.

Although $\mathrm{Cr}$ and $\mathrm{Ni}$ can probably also dissolve in this solid solution, this was not considered in the present model because of lack of data and because good results were obtained in most cases without this. 


\subsection{Optimization of CVS liquidus calculations}

In many cases, interactions between minor constituents of the CVS glasses can be important. For example, an $w_{\text {zsiay }}$ ternary parameter is necessary in the calculations. However this parameter cannot be obtained by simply optimizing data for the $\mathrm{ZrO}_{2}-\mathrm{SiO}_{2}-\mathrm{Al}_{2} \mathrm{O}_{3}$ ternary system because $\mathrm{ZrO}_{2}$ and $\mathrm{Al}_{2} \mathrm{O}_{3}$ are minor constituents of the glasses, and the interactions between these constituents in a predominantly sodium borosilicate glass is quite different from that in a $\mathrm{ZrO}_{2}-\mathrm{SiO}_{2}-\mathrm{Al}_{2} \mathrm{O}_{3}$ glass. Consequently, it is only possible to optimize such parameters from actual measurements on the CVS glasses. In this way the following parameters were determined:

$$
\begin{aligned}
& \omega_{\text {zssi (A) }}^{01}=-313.80 \mathrm{~kJ} / \mathrm{mol} \\
& \omega_{\text {Lisi (Zi) }}^{29}=-138.07 \mathrm{~kJ} / \mathrm{mol} \\
& \omega_{\text {Casi (日) }}^{01}=62.76 \mathrm{~kJ} / \mathrm{mol} \\
& \omega_{\text {ESi (z) }}^{01}=-12.55 \mathrm{~kJ} / \mathrm{mol} \\
& \dot{\omega} \stackrel{1}{\text { isi (cs) }}=-100.42 \mathrm{~kJ} / \mathrm{mol} \\
& \omega_{\text {Mosi (z) }}^{\text {il }}=167.36 \mathrm{~kJ} / \mathrm{mol} \\
& \omega_{\text {Casi }[2]}=-41.84 \mathrm{~kJ} / \mathrm{mol} \\
& \omega_{\text {casi }}^{01} \text { (Fo) }=-87.86 \mathrm{~kJ} / \mathrm{mol} \\
& \omega_{\text {Fosi (Mol }}^{\text {or }}=16.74 \mathrm{~kJ} / \mathrm{mol}
\end{aligned}
$$

\section{INCLUDING Fe $\mathrm{e}^{2+}$ IN THE DATABASES}

Optimizations were performed for the liquid and sollid phases of the $\mathrm{FeO}-\mathrm{Fe}_{2} \mathrm{O}_{3}-\mathrm{SiO}_{2}-\mathrm{CaO}$ system. An article on this work is in preparation. The following systems involving $\mathrm{Fe}^{2+}$ were also optimized: $\mathrm{FeO}-\mathrm{MgO}, \mathrm{FeO}-\mathrm{ZrO}_{2}, \mathrm{FeO}-\mathrm{Al}_{2} \mathrm{O}_{3}-\mathrm{SiO}_{2}$. All these optimizations will appear in "Phase Diagrams for Ceramists". No data for the FeO- $\mathrm{B}_{2} \mathrm{O}_{3}$ system could be found, so ideal mixing was assumed.

The optimized parameters have been incorporated into the database for the glass and can be used to calculate the effect of oxygen pressure on the $\mathrm{Fe}^{2+} / \mathrm{Fe}^{3+}$ ratio in the glass (using the EQUILIB program and the ACT option).

$\mathrm{Fe}^{2+}$ has also been incorporated into the database for the spinel phase as discussed in Section 8.

Data for $\mathrm{Fe}^{2+}$ in solid crystalline phases and solid solutions such as $\mathrm{Fe}_{2} \mathrm{SiO}_{4}-\mathrm{M}_{2} \mathrm{SiO}_{4}$ or $\mathrm{FeSiO}_{3}-\mathrm{MSiO}_{3}$ solutions $(\mathrm{M}=\mathrm{Mg}$, Ca) were also optimized, but these phases were never 
found to form as primary phases from the CVS glasses in the calculations and so they have not been included in the database prepared for Battelle.

\section{INCLUDING CHROME IN THE DATABASES}

Most binary, ternary and quaternary sub-systems of the $\mathrm{CrO}-\mathrm{Cr}_{2} \mathrm{O}_{3}-\mathrm{SiO}_{2}-\mathrm{CaO}-\mathrm{Al}_{2} \mathrm{O}_{3}-\mathrm{FeO}-\mathrm{Fe}_{2} \mathrm{O}_{3}$ system have been fully optimized. Two articles are being prepared based on this work. As well; the optimizations will appear in "Phase Diagrams for Ceramists". Good quantitative reproduction of all phase diagram and thermodynamic data has been obtained, including the effect of $\mathrm{O}_{2}$ pressure on the $\mathrm{Cr}^{2+} / \mathrm{Cr}^{3+}$ ratio and on the phase equilibria.

Data for several $\mathrm{Cr}$-containing solid phases were also optimized. The spinel phase is discussed in Section 8. A database was prepared for the corundum solid solution, $\mathrm{Fe}_{2} \mathrm{O}_{3}-\mathrm{Al}_{2} \mathrm{Q}_{3}-\mathrm{Cr}_{2} \mathrm{O}_{3}$. This phase was. found in the calculations to be the primary phase for certain CVS glasses. None of the other Cr-containing solid phases were found to form as primary phases from the CVS glasses in the calculations, and so they have not been included in the database prepared for Battelle.

No data for the $\mathrm{Na}_{2} \mathrm{O}-\mathrm{CrO}-\mathrm{Cr}_{2} \mathrm{O}_{3}$ system were found. Consequently, one parameter $\omega_{\mathrm{NoCr}(3+1}$ was optimized by using spinel liquidus data for the CVS glasses.

$\mathrm{Cr}^{8+}$ has not been included in the database due to lack of available data. This oxidation state will be most important in basic glasses.

\section{$5 \quad$ INCLUDING NICKEL IN THE DATABASES}

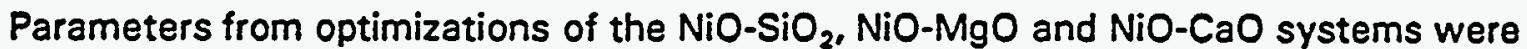
included in the glass database. These optimisations are to be published in "Phase Diagrams for Ceramists". The phase $\mathrm{Ni}_{2} \mathrm{SiO}_{4}$ was optimized but was found never to appear as a primary phase in the calculaions. Consequently, it has not been included in the database prepared for Battelle. No data for the $\mathrm{Na}_{2} \mathrm{O}-\mathrm{B}_{2} \mathrm{O}_{3}-\mathrm{NiO}$ system or its sub-systems were found. Hence, an $\omega_{\text {NWN }}$ and an $\omega_{\text {BN }}$ parameter were optimized by using spinel liquidus data for the CVS glasses. The spinel phase, which contains nickel, is discussed in Section 8.

\section{INCLUDING MANGANESE IN THE DATABASES}

Parameters obtained by optimizing the following systems were included in the glass database: $\mathrm{MgO}-\mathrm{MgO}, \mathrm{MnO}-\mathrm{SiO}_{2}, \mathrm{MnO}-\mathrm{CaO}, \mathrm{MnO}-\mathrm{Al}_{2} \mathrm{O}_{3}$, and $\mathrm{MnO}-\mathrm{ZrO}_{2}$. These optimizations will appear in "Phase Diagrams for Ceramists". From an examination of available data it was 
concluded that $\mathrm{Mn}^{3+}$ will not appear in appreciable concentrations in CVS glasses, and so $\mathrm{Mn}^{3+}$ was not included in the glass database.

Data for the $\mathrm{MnSiO}_{3}$ solid phase and $\mathrm{Mn}_{2} \mathrm{SiO}_{4}-\mathrm{Mg}_{2} \mathrm{SiO}_{4}$ solid solutions were also included in Battelle's databases. Although neither of these phases appeared in the calculations for the CVS glasses, they could appear in glasses with somewhat higher $\mathrm{Mn}$ contents.

Manganese has been included in the spinel phase as discussed in Section 8.

\section{CALCULATIONS FOR CVS GLASSES}

Experiments on 123 compositions were carried out by Battelle. The compositions are listed in Table 5. The ID number is that used by Battelle. Glasses 1 to 23 are CVS-I. Glasses 24 to 123 are CVS-ll glasses 1 to 100. The compositions of "others" is shown in Table 6 as regards $\mathrm{Cr}, \mathrm{Ni}$ and $\mathrm{Mn}$ content.

Experimental liquidus temperatures, primary phases and the elements observed by SEM/EDS in the primary phases are shown in Table 7 which is reproduced from the most recent tables supplied to us by Battelle in 1994.

Calculated liquidus temperatures and primary phases are shown in Table 8. These were calculated by assuming that no prior precipitation of spinel occurs (that is, it is assumed that the amount of any prior spinel precipitation has a negligible effect on the glass composition). For some glass compositions, the calculations indicated that two immiscible glass phases exist at equilibrium. However, the calculations shown in Table 8 were all performed by assuming only one glass phase. In cases where two glass phases appear at equilibrium, taking account of this fact in the calculations usually only changes the calculated liquidus temperature by $10^{\circ}$ or less. In cases where two "primary" phases appear in Table 8 , the lower temperature liquidus is a metastable liquidus calculated by suppressing the formation of the phase appearing at the higher temperature.

Note that in Table 8 the "CO(R)" phase is the corundum solid solution $\left(\mathrm{Fe}_{2} \mathrm{O}_{3}-\mathrm{Al}_{2} \mathrm{O}_{3}-\mathrm{Cr}_{2} \mathrm{O}_{3}\right)$. Clinopyroxene $(\mathrm{Cp})$ is the $\mathrm{NaFeSi}_{2} \mathrm{O}_{6}-\mathrm{CaMgSi}_{2} \mathrm{O}_{6}$ solid solution.

Calculations of the spinel liquidus, also shown in Table 8, are discussed in Section 8.

The amounts of $\mathrm{Ni}, \mathrm{Cr}$ and $\mathrm{Mn}$ in the "others" (as in Table 6) was taken into account in the calculations. The remaining "others" were grouped together as the one fictitious component " $\mathrm{In}_{2} \mathrm{O}_{3}$ " as described in the earlier report [92 Pel]. (This "compound" is included in the glass database but in no solid phases. Its affect is only to lower the activities of the other components. $\ln _{2} \mathrm{O}_{3}$ was chosen because its molecular weight is close to the average of the "others"). 
Table 5

Compositions of CVS-1 and CVS-II Glasses

\begin{tabular}{|c|c|c|c|c|c|c|c|c|c|c|}
\hline \multicolumn{10}{|c|}{ Composition (weight fraction) } & \multirow{2}{*}{$\frac{\text { ID }}{\text { No }}$} \\
\hline $\mathrm{siO}_{2}$ & $\mathrm{~B}_{2} \mathrm{O}_{3}$ & $\mathrm{Na}_{2} \mathrm{O}$ & $\mathrm{Ii}_{2} \mathrm{O}$ & $\mathrm{CaO}$ & Mgo & $\mathrm{Fe}_{2} \mathrm{O}_{3}$ & $\mathrm{Al}_{2} \mathrm{O}_{3}$ & $2 \Omega_{3}$ & Others & \\
\hline .4801 & .2142 & .1003 & .0376 & .0275 & .0363 & .0568 & .0636 & .0429 & .0407 & 1 \\
\hline .5500 & .0500 & .0500 & .0700 & .1000 & .0000 & $.0200^{\circ}$ & .1500 & .0000 & .0100 & 2 \\
\hline .4200 & .2000 & .0500 & .0700 & .0000 & .0800 & .0200 & .1400 & .0100 & .0100 & 3 \\
\hline .5700 & .2000 & .0900 & .0100 & .0200 & .0800 & .0200 & .0000 & .0000 & .0100 & 1 \\
\hline .5700 & .0500 & .0700 & .0700 & .0000 & .0000 & .1500 & .0800 & .0000 & .0100 & 5 \\
\hline .4400 & .2000 & .0500 & .0700 & .0000 & .0000 & .0200 & .0000 & .1200 & .1000 & 6 \\
\hline .5700 & .0500 & .0964 & .0100 & .1000 & .0000 & .0336 & .0000 & .1300 & .0100 & 7 \\
\hline .5363 & .0500 & .0837 & .0100 & .0000 & .0800 & .1500 & .0000 & .0800 & .0100 & 8 \\
\hline .4200 & .1962 & .0538 & .0100 & .0000 & .0800 & .1400 & .0000 & .0000 & .1000 & 9 \\
\hline .5700 & .0851 & .0949 & .0100 & .0000 & .0000 & .0200 & .1200 & .0000 & .1000 & 10 \\
\hline .4200 & .2549 & .0751 & .0100 & .1000 & .0000 & .0200 & .1400 & .0000 & .0800 & 11 \\
\hline .4200 & .1764 & .0736 & .0700 & .1000 & .0000 & .1500 & .0000 & .0000 & .0100 & 22 \\
\hline .5700 & .2000 & .1862 & .0100 & .0000 & .0000 & .0200 & .0038 & .0000 & .0100 & 13 \\
\hline .4200 & .2000 & .1862 & .0100 & .0000 & .0000 & .0200 & .0238 & .1300 & .0100 & 14 \\
\hline .5589 & .0500 & .1211 & .0700 & .0000 & .0800 & .0200 & .0000 & .0000 & .1000 & 25 \\
\hline .4327 & .0500 & .1873 & .0100 & .0000 & .0800 & .0858 & .1442 & .0000 & .0100 & 16 \\
\hline .4545 & .0500 & .1455 & .0100 & .1000 & .0000 & .1400 & .0000 & .0000 & .1000 & 17 \\
\hline .4214 & .0500 & .1186 & .0700 & .0200 & .0800 & .0200 & .0000 & .1300 & .0900 & 18 \\
\hline .4801 & .1142 & .1003 & .0376 & .0275 & .0363 & .0568 & .0636 & .0429 & .0407 & 19 \\
\hline .4801 & .1142 & .1003 & .0376 & .0275 & .0363 & .0568 & .0636 & .0429 & .0407 & 20 \\
\hline .5700 & .2000 & .0900 & .0100 & .0200 & .0800 & .0200 & .0000 & .0000 & .0100 & 21 \\
\hline .5363 & .0500 & .0837 & .0100 & .0000 & .0800 & .1500 & .0000 & .0800 & .0100 & 22 \\
\hline .5153 & .0956 & .1052 & .0375 & .0289 & .0084 & .1179 & .0456 & .0063 & .0393 & 23 \\
\hline .5226 & .0874 & .0700 & .0600 & .0000 & .0500 & .0400 & .0800 & .0100 & .0800 & 24 \\
\hline .5017 & .0700 & .0883 & .0600 & .0700 & .0000 & .0450 & .1100 & .0300 & .0250 & 25 \\
\hline
\end{tabular}




\section{Table 5 (continued)}

\section{Compositions of CVS-I and CVS-11 Glasses}

\begin{tabular}{|c|c|c|c|c|c|c|c|c|c|c|}
\hline \multicolumn{10}{|c|}{ composition (weight fraction) } & ID \\
\hline $\mathrm{Sia}_{3}$ & $\mathrm{~B}_{2} \mathrm{O}_{3}$ & $\mathrm{Na}_{2} \mathrm{O}$ & $\mathrm{Ii}_{2} \mathrm{O}$ & $\mathrm{CaO}$ & $\mathrm{Mg}$ & $\mathrm{Fe}_{2} \mathrm{O}_{3}$ & $\mathrm{Al}_{2} \mathrm{O}_{3}$ & $2 \mathrm{IO}_{3}$ & Others & No \\
\hline .4645 & .1320 & .0700 & .0435 & .0700 & .0100 & .0450 & .1032 & .0368 & .0250 & 26 \\
\hline .5600 & .1095 & .0700 & .0536 & .0700 & .0000 & .0400 & $.0619^{\circ}$ & .0100 & .0250 & 27 \\
\hline .4751 & .1590 & .1010 & .0200 & .0348 & .0000 & .0400 & .0800 & .0100 & .0800 & 28 \\
\hline .5373 & .0700 & .0700 & .0382 & .0700 & .0046 & .1200 & .0159 & .0100 & .0641 & 29 \\
\hline .4814 & .1700 & .0700 & .0591 & .0094 & .0000 & .0400 & .0953 & .0100 & .0648 & 30 \\
\hline .5115 & .0700 & .0985 & .0600 & .0000 & .0500 & .1140 & .0610 & .0100 & .0250 & 31 \\
\hline .5431 & .0944 & .0924 & .0600 & .0000 & .0000 & .0712 & .0138 & .1000 & .0250 & 32 \\
\hline .4694 & .1700 & .1306 & .0200 & .0000 & .0000 & .0669 & .1043 & .0100 & .0288 & 33 \\
\hline .4915 & .0751 & .0833 & .0600 & .0700 & .0100 & .0400 & .0100 & .0935 & .0665 & 34 \\
\hline .4683 & .1700 & .0700 & .0466 & .0700 & .0100 & .0400 & .0901 & .0100 & .0250 & 35 \\
\hline .4937 & .0700 & .1692 & .0225 & .0300 & .0500 & .0400 & .0896 & .0100 & .0250 & 36 \\
\hline .4600 & .1313 & .0802 & .0486 & .0500 & .0200 & .0400 & .0243 & .2000 & .0457 & 37 \\
\hline .4729 & .0700 & .1700 & .0214 & .0601 & .0000 & .0400 & .0756 & .0100 & .0800 & 38 \\
\hline .5353 & .1053 & .1 & .0375 & .0083 & .0084 & .0719 & .0231 & .0385 & .0592 & 39 \\
\hline .4801 & .1142 & .1003 & .0376 & .0275 & .0363 & 68 & .0636 & .0429 & .0407 & 40 \\
\hline .5353 & .1053 & .1125 & .0375 & .0083 & .0084 & .0719 & .0231 & .0385 & .0592 & 11 \\
\hline .5328 & .1048 & .1129 & .0373 & .0082 & .0084 & .0733 & .0235 & .0392 & .0596 & 12 \\
\hline .5700 & .0500 & .1031 & .0669 & .0000 & .0000 & .0600 & .0100 & .1300 & .0100 & 13 \\
\hline .5700 & .1314 & .0500 & .0700 & .0000 & .0800 & .0200 & .0686 & .0000 & .0100 & 41 \\
\hline .5700 & .0500 & .0735 & .0700 & .0000 & .0800 & .0200 & .0365 & .0000 & .1000 & 45 \\
\hline .5700 & .0522 & .2000 & .0100 & .0800 & .0000 & .0200 & .0578 & .0000 & .0100 & 46 \\
\hline .4464 & .2000 & .0736 & .0700 & .0000 & .0000 & .0200 & .0961 & .0000 & .0939 & 47 \\
\hline .5059 & .0500 & .0841 & .0700 & .0800 & .0000 & .1500 & .0033 & .0000 & .0567 & 48 \\
\hline .4431 & .2000 & .0512 & .0700 & .0800 & .0000 & .0200 & .0257 & .1000 & .0100 & 49 \\
\hline .5463 & .0500 & .2000 & .0155 & .0000 & .0800 & .0200 & .0782 & .0000 & .0100 & 50 \\
\hline .5619 & .0500 & .2000 & .0126 & .0000 & .0000 & .0200 & .0555 & .0000 & .1000 & 51 \\
\hline .4391 & .2000 & .0675 & .0100 & .0800 & .0000 & .0200 & .0000 & .0834 & .1000 & 52 \\
\hline .5190 & .2000 & .0832 & .0100 & .0000 & .0000 & .1320 & .0458 & .0000 & .0100 & 53 \\
\hline
\end{tabular}




\section{Table 5 (continued)}

\section{Compositions of CVS-I and CVS-II Glasses}

\begin{tabular}{|c|c|c|c|c|c|c|c|c|c|c|}
\hline \multicolumn{10}{|c|}{ Composition (weight fraction) } & \multirow{2}{*}{ ID } \\
\hline sis & $\mathrm{B}_{2} \mathrm{O}_{3}$ & $\mathrm{Na}_{2} \mathrm{O}$ & $\mathrm{Ii}_{2} \mathrm{O}$ & $\mathrm{CaO}$ & $\mathrm{Mg}$ & $\mathrm{FeO}_{3}$ & $\mathrm{Al}_{2} \mathrm{O}_{3}$ & 2103 & others & \\
\hline .5700 & .1843 & .0500 & .0331 & .0800 & $.0000^{\circ}$ & .0200 & .0526 & .0000 & .0100 & 51 \\
\hline .5445 & .0500 & .2000 & .0428 & .0000 & .0000 & .0200 & .0027 & .1300 & .0100 & 55 \\
\hline .4200 & .0544 & .2000 & .0364 & .0000 & .0800 & .0200 & .0892 & .0000 & .1000 & 56 \\
\hline .4200 & .1743 & .2000 & .0369 & .0000 & .0000 & .0200 & .1388 & .0000 & .0100 & 57 \\
\hline .4200 & .0500 & .2000 & .0428 & .0800 & .0000 & .0632 & .1340 & .0000 & .0100 & 58 \\
\hline .5421 & .0500 & .0891 & .0700 & .0800 & .0000 & .1500 & .0088 & .0000 & .0100 & 59 \\
\hline .5700 & .0839 & .1061 & .0700 & .0000 & .0000 & .0200 & .1400 & .0000 & .0100 & 60 \\
\hline .5147 & .1109 & .1044 & .0100 & .0000 & .0800 & .1428 & .0272 & .0000 & .0100 & 61 \\
\hline .4838 & .0500 & .1362 & .0700 & .0000 & .0800 & .0742 & .0258 & .0700 & .0100 & 62 \\
\hline .5040 & .0639 & .1500 & .0421 & .0200 & .0500 & .0200 & .1000 & .0200 & .0300 & 63 \\
\hline .5325 & .0694 & .0781 & .0700 & .0500 & .0200 & .0300 & .1000 & .0200 & .0300 & 64 \\
\hline .5675 & .0500 & .0625 & .0700 & .0320 & .0380 & .1000 & .0300 & .0200 & .0300 & 65 \\
\hline .5070 & .1477 & .0500 & .0653 & .0200 & .0300 & .0300 & .0500 & .0700 & .0300 & 66 \\
\hline .5700 & .1078 & .0500 & .0699 & .0500 & .0200 & .0200 & .0623 & .0200 & .0300 & 67 \\
\hline .5299 & .2106 & .0500 & .0595 & .0200 & .0500 & .0308 & .0592 & .0200 & .0700 & 68 \\
\hline .5264 & .1259 & .0577 & .0700 & .0200 & .0200 & .0200 & .0746 & .0200 & .0654 & 69 \\
\hline .5294 & .0500 & .2277 & .0429 & .0500 & .0200 & .0200 & .0400 & .0500 & .0700 & 70 \\
\hline .4700 & .1442 & .0968 & .0390 & .0500 & .0200 & .0200 & .0854 & .0200 & .0546 & 71 \\
\hline .5073 & .1357 & .0957 & .0413 & .0200 & .0200 & .0515 & .0785 & .0200 & .0300 & 72 \\
\hline .4801 & .1142 & .1003 & .0376 & .0275 & .0363 & .0568 & .0636 & .0429 & .0407 & 73 \\
\hline .5328 & .2048 & .1129 & .0373 & .0082 & .0084 & .0733 & .0235 & .0392 & .0596 & 71 \\
\hline .6000 & .0817 & .0450 & .0788 & .0008 & .0009 & .0720 & .0233 & .0385 & .0590 & 75 \\
\hline .5226 & .0874 & .0700 & .0600 & .0000 & .0500 & .0400 & .0800 & .0100 & .0800 & 76 \\
\hline .5328 & .1048 & .1129 & .0373 & .0082 & .0084 & .0733 & .0235 & .0392 & .0596 & 77 \\
\hline .5328 & .1048 & .1229 & .0373 & .0082 & .0084 & .0733 & .0235 & .0392 & .0596 & 78 \\
\hline .5328 & .1048 & .1129 & .0373 & .0082 & .0084 & .0733 & .0235 & .0392 & .0596 & 79 \\
\hline .5328 & .1048 & .1129 & .0373 & .0082 & .0084 & .0733 & .0235 & .0392 & .0596 & 80 \\
\hline
\end{tabular}


Table 5 (continued)

Compositions of CVS-I and CVS-II Glasses

\begin{tabular}{|c|c|c|c|c|c|c|c|c|c|c|}
\hline \multicolumn{10}{|c|}{ Carmosition (weight fraction) } & ID \\
\hline sio, & $\mathrm{B}_{2} \mathrm{O}_{3}$ & $\mathrm{Na}_{2} \mathrm{O}$ & $\mathrm{Ii}_{2} \mathrm{O}$ & $\mathrm{CaO}$ & $\mathrm{MgO}$ & $\mathrm{Fe}_{2} \mathrm{O}_{3}$ & $\mathrm{Al}_{2} \mathrm{O}_{3}$ & $\mathrm{ZrO}$ & Others & No \\
\hline .3900 & .2000 & .0500 & .0700 & .0200 & .0800 & .0200 & .1500 & .0100 & .0100 & 81 \\
\hline .4380 & .2718 & .1268 & .0727 & .0375 & .0005 & .0200 & .1150 & .0075 & .0102 & 82 \\
\hline .5281 & .0876 & .1725 & .0743 & .0063 & .0005 & .0200 & .0925 & .0075 & .0107 & 83 \\
\hline .5281 & .0664 & .2200 & .0730 & .0000 & .0000 & .0200 & .1625 & .0175 & .0125 & 81 \\
\hline .5579 & .1765 & .1125 & .0156 & .0500 & .0005 & .0200 & .0500 & .0075 & .0095 & 85 \\
\hline .3232 & .1717 & .1900 & .0051 & .1000 & .0000 & .0200 & .1800 & .0000 & .0100 & 86 \\
\hline .5697 & .0509 & .0925 & .0642 & .0025 & .0008 & .0812 & .0288 & .0431 & .0663 & 87 \\
\hline .5344 & .1228 & .0860 & .0697 & .0007 & .0004 & .0013 & .0196 & .1548 & .0203 & 88 \\
\hline .5175 & .0917 & .1211 & .0523 & .0097 & .0061 & .0388 & .1180 & .0026 & .0422 & 89 \\
\hline .4596 & .1587 & .1086 & .0583 & .0024 & .0001 & .0004 & .2043 & .0000 & .0076 & 90 \\
\hline .5040 & .1355 & .0797 & .0696 & .0007 & .0002 & .0046 & .1640 & .0001 & .0416 & 92 \\
\hline .5660 & .0781 & .0664 & .0713 & .0079 & .0032 & .0334 & .0816 & .0005 & .0916 & 92 \\
\hline .4854 & .1418 & .0812 & .0691 & .0008 & .0008 & .0080 & .1819 & .0005 & .0305 & 93 \\
\hline .5697 & .0509 & .0925 & .0642 & .0025 & .0008 & .0812 & .0288 & .0431 & .0663 & 94 \\
\hline .5175 & .0917 & .1211 & .0523 & .0097 & .0061 & .0388 & .1180 & .0026 & .0422 & 95 \\
\hline .5040 & .1355 & .0797 & .0696 & .0007 & .0002 & .0046 & .1640 & .0001 & .0416 & 96 \\
\hline .5660 & .0781 & .0664 & .0713 & .0079 & .0032 & .0334 & .0816 & .0005 & .0916 & 97 \\
\hline .4854 & .1418 & .0812 & .0691 & .0008 & .0008 & .0080 & .1819 & .0005 & .0305 & 98. \\
\hline .5018 & .0600 & .1800 & .0632 & .0400 & .0050 & .1050 & .0200 & .0050 & .0200 & 99 \\
\hline .4550 & .0600 & .1800 & .0700 & .0050 & .0050 & .0050 & .0200 & .1100 & .0900 & 100 \\
\hline .5600 & .1600 & .0500 & .0254 & .0050 & .0400 & .0699 & .0200 & .0497 & .0200 & 101 \\
\hline .5479 & .1600 & .0500 & .0121 & .0050 & .0050 & .1050 & .0200 & .0050 & .0900 & 102 \\
\hline .5074 & .1600 & .0500 & .0176 & .0050 & .0400 & .1050 & .0200 & .0750 & .0200 & 103 \\
\hline .4400 & .0600 & .1734 & .0700 & .0050 & .0400 & .1050 & .0200 & .0050 & .0816 & 204 \\
\hline .5600 & .0950 & .1800 & .0700 & .0050 & .0400 & .0050 & .0200 & .0050 & $.02,00$ & 105 \\
\hline .4900 & .0951 & .1800 & .0699 & .0400 & .0050 & .0050 & .0200 & .0050 & .0900 & 106 \\
\hline
\end{tabular}


Table 5 (continued)

Compositions of CVS-1 and CVS-11 Glasses

\begin{tabular}{|c|c|c|c|c|c|c|c|c|c|c|}
\hline \multicolumn{10}{|c|}{ Composition (weight fraction) } & \multirow{2}{*}{$\frac{\text { ID }}{\text { No }}$} \\
\hline sig & $\mathrm{B}_{2} \mathrm{O}_{3}$ & $\mathrm{Na}_{2} \mathrm{O}$ & $\mathrm{Ii} \mathrm{O}$ & CaO & $\mathrm{MgO}$ & $\mathrm{Fe}_{3}$ & $\mathrm{~A}_{2} \mathrm{O}_{3}$ & $\mathrm{ZrO}_{2}$ & Others & \\
\hline .4550 & .0600 & .1800 & .0700 & .0050 & .0050 & .1050 & .0200 & .0800 & .0200 & 207 \\
\hline .4400 & .0600 & .1800 & .0700 & .0050 & .0200 & .0050 & .1700 & .0050 & .0450 & 208 \\
\hline .4764 & .0600 & .1800 & .0136 & .0400 & .0050 & .0050 & .1700 & .0050 & .0450 & 109 \\
\hline .4983 & .0800 & .1800 & .0180 & .0137 & .0050 & .0250 & .0987 & .0613 & .0200 & 110 \\
\hline .4597 & .0 & 3 & .1 & .0 & .0050 & .0250 & .1050 & .0750 & .0200 & 111 \\
\hline .4400 & .1171 & .1800 & .0100 & .0400 & .0050 & 50 & .0200 & .0629 & 00 & 112 \\
\hline .5600 & .1600 & .0542 & .0700 & .0050 & .0050 & .1008 & .0200 & .0050 & .0200 & 113 \\
\hline .5600 & .1600 & .1050 & .0100 & .0050 & .0400 & .0050 & .0200 & .0050 & .0900 & 114 \\
\hline .4400 & .1600 & .1000 & .0700 & .0050 & .0400 & .0050 & .0200 & .0700 & .0900 & 215 \\
\hline .4400 & .1337 & .1279 & .0700 & .0098 & .0050 & .0986 & .0200 & .0050 & .0900 & 116 \\
\hline .4400 & .1600 & .1800 & .0526 & .0400 & .0050 & .0271 & .0703 & .0050 & .0200 & 117 \\
\hline .463 & .109 & .263 & .042 & .010 & .016 & .087 & .032 & .004 & .074 & 118 \\
\hline .4801 & .1142 & .1003 & .0376 & .0275 & .0363 & .0568 & .0636 & .0429 & .0407 & 119 \\
\hline .5328 & .1048 & .1129 & .0373 & .0082 & .0084 & .0733 & .0235 & .0392 & .0596 & 120 \\
\hline. .4200 & .1743 & .2000 & .0369 & .0000 & .0000 & .0200 & .1388 & .0000 & .0100 & 121 \\
\hline .5203 & .0969 & .0980 & .0356 & .0097 & .0077 & .1019 & .0523 & .0199 & .0577 & 122 \\
\hline .5329 & .0740 & .0626 & .0596 & .0035 & .0012 & .1229 & .0286 & .0443 & .0704 & 123 \\
\hline & & & & & & & & & & \\
\hline
\end{tabular}




\section{Table 6}

Compositions of "Others" in CVS Glasses

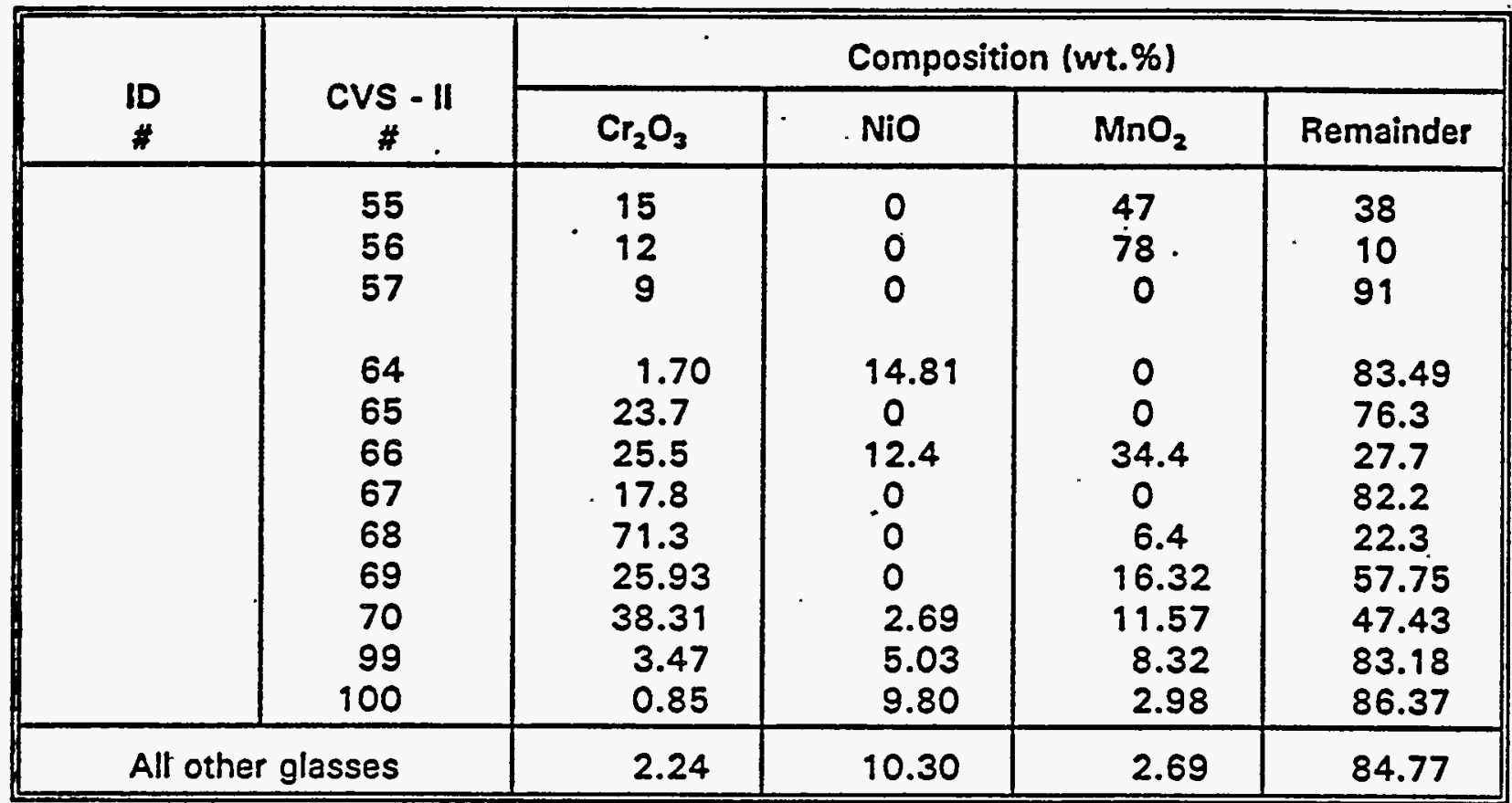




\begin{tabular}{|c|c|c|c|c|}
\hline $\begin{array}{c}\text { CVS } \\
n\end{array}$ & $\begin{array}{c}\text { Experimental } \\
\text { TI phase }\end{array}$ & $\begin{array}{l}\text { SEMVEDS } \\
\text { olements }\end{array}$ & TI Spinel & $\begin{array}{c}\text { no spinel } \\
T \text { range }\end{array}$ \\
\hline$T$ & $\begin{array}{l}950 \mathrm{ZS} \\
003 \mathrm{Cp}\end{array}$ & & 977 & \\
\hline 2 & $\begin{array}{l}927 \mathrm{CS} \\
916 \mathrm{LA}\end{array}$ & $\begin{array}{l}\mathrm{Ca}, \mathrm{SI} \\
\mathrm{Al}, \mathrm{SI}\end{array}$ & & 891.1038 \\
\hline$\overline{3}$ & $929 \mathrm{OV}$ & $\mathrm{Mg}, \mathrm{Sl} ; \mathrm{Fo}, \mathrm{Ni}$ & & $868-1038$ \\
\hline$\overline{4}$ & 957 SO & SI & & $927-1038$ \\
\hline 5 & $939 \mathrm{Op}$ & $F_{0, S 1}$ & 1035 & \\
\hline 6 & 2111825 & & & $1014-1118$ \\
\hline 7 & $\begin{array}{l}>1118 \mathrm{ZS} \\
>1118 \mathrm{CZ}\end{array}$ & & & $1074-1118$ \\
\hline$\overline{8}$ & $\begin{array}{l}>1118 \mathrm{ZS} \\
>1118 \text { Op }\end{array}$ & $\begin{array}{l}\mathrm{Zr}, \mathrm{Sl} \\
\mathrm{Mg}, \mathrm{Sl}\end{array}$ & $>1118$ & \\
\hline 9 & $1097(?)$ & $\mathrm{Mg}, \mathrm{F}_{\theta}, \mathrm{Sl} ; \mathrm{Cr}, \mathrm{NI}$ & $>1118$ & \\
\hline 10 & \multicolumn{2}{|c|}{ (7) (?), severe segregation } & $>1118$ & \\
\hline 11 & $919 \mathrm{CS}$ & $\mathrm{Ca}, \mathrm{Sl} ; \mathrm{Nd}, \mathrm{La}$ & 1035 & \\
\hline 12 & $8260 p$ & Fo,Si & & $752-1038$ \\
\hline 13 & $<571$ (Non $\theta)$ & & & $571 \cdot 1038$ \\
\hline 14 & $<571$ (Nono) & & & 571.1038 \\
\hline 15 & $866 \mathrm{OV}$ & $\mathrm{Mg} \mathrm{NI}_{1} \mathrm{Sl}_{i} \mathrm{Cr}$ & & 837.1038 \\
\hline 16 & $\$ 1118$ OV & $\mathrm{Mg}, \mathrm{SI}$ & $>1118$ & \\
\hline 17 & $\begin{array}{l}943 \mathrm{CS} \\
813 \mathrm{Cp}\end{array}$ & $\begin{array}{l}\mathrm{Ca}, \mathrm{SI} \\
\mathrm{Ca}, \mathrm{Fo}, \mathrm{Sl} ; \mathrm{Na}, \mathrm{NI}\end{array}$ & 1000 & \\
\hline 18 & 2111820 & $\mathrm{Zr}$ & & $1014-1118$ \\
\hline 19 & $\begin{array}{l}916 \mathrm{ZS} \\
016 \mathrm{Cp}\end{array}$ & $\mathrm{Ca}_{1} \mathrm{Mg}, \mathrm{Fo}, \mathrm{Sl} ; \mathrm{NI}, \mathrm{Cr}$ & 961 & \\
\hline 20 & $\begin{array}{l}913 \mathrm{ZS} \\
913 \mathrm{Cp}\end{array}$ & & 966 & \\
\hline 21 & 85750 & & & 927.1038 \\
\hline 22 & $\begin{array}{l}>1118 \text { ZS } \\
>1118 \text { Op }\end{array}$ & & $>1118$ & \\
\hline 23 & $858 \mathrm{Cp}$ & & 980 & \\
\hline 1 & 929 Ov & $\mathrm{Mg}, \mathrm{NI}, \mathrm{Sl}_{;} \mathrm{Fo}_{0} \mathrm{Cr}$ & 1048 & \\
\hline 2 & $\begin{array}{l}830 \mathrm{CS} \\
800 \mathrm{Cp}\end{array}$ & $\begin{array}{l}\mathrm{Ca}, \mathrm{SI} \\
\mathrm{Ca}, \mathrm{Fo}_{\mathrm{O}} \mathrm{NI}, \mathrm{SI}\end{array}$ & . & $826 \cdot 928$ \\
\hline
\end{tabular}

\begin{tabular}{|c|c|c|c|c|}
\hline $\begin{array}{c}\text { CVS } \\
n\end{array}$ & $\begin{array}{c}\text { Experimental } \\
\text { TI phaso }\end{array}$ & $\begin{array}{l}\text { SEMEDS } \\
\text { olements }\end{array}$ & TI Spinel & $\begin{array}{l}\text { no spinel } \\
\text { T range }\end{array}$ \\
\hline 3 & $\begin{array}{l}831 \mathrm{ZS} \\
887 \mathrm{Cp}\end{array}$ & & & $782 \cdot 1038$ \\
\hline 4 & $\begin{array}{l}810 \mathrm{CS} \\
800 \mathrm{Cp}\end{array}$ & $\begin{array}{l}\mathrm{Ca}, \mathrm{Si} \\
\mathrm{Ca}, \mathrm{F} \theta, \mathrm{NI}_{1} \mathrm{Si}\end{array}$ & & $702-812$ \\
\hline 5 & $832 \mathrm{CS}$ & Ca,Si;Zr,Nd,La & 888 & \\
\hline 6 & $939 \mathrm{Cp}$ & $\mathrm{Ca}, \mathrm{Fe}_{\mathrm{S}} \mathrm{Si} ; \mathrm{NI}, \mathrm{Cr}$ & $>1004$ & \\
\hline 7 & $819 \mathrm{Cp}$ & $\mathrm{Ca}, \mathrm{Fo}, \mathrm{NI}, \mathrm{SI}$ & 934 & \\
\hline 8 & 866 Op & $\mathrm{Fe}_{\theta}, \mathrm{Sl}_{i} \mathrm{Mg}$ & 21118 & \\
\hline 9 & $1018 \mathrm{ZS}$ & & & $\overline{735-1146}$ \\
\hline 10 & $<585$ (None) & & $>868$ & \\
\hline 11 & $1035 \mathrm{ZS}$ & $\mathrm{Zr}, \mathrm{Si}$ & & $927-1038$ \\
\hline 12 & $840 \mathrm{Cp}$ & $\mathrm{Ca}, \mathrm{F}_{\theta}, \mathrm{Mg}, \mathrm{Si}_{i} \mathrm{Ni}, \mathrm{Cr}$ & $\div$ & 826.918 \\
\hline 13 & $909 \mathrm{Cp}$ & $\mathrm{Ca}, \mathrm{F} \theta, \mathrm{Mg}, \mathrm{SI}$ & & $826-911$ \\
\hline 14 & $>111825$ & & & $1014-1118$ \\
\hline 15 & $<679$ (None) & & $\overline{956}$ & \\
\hline 16 & $877 \mathrm{Cp}$ & & 906 & \\
\hline 17. & $\begin{array}{l}925 \mathrm{ZS} \\
925 \mathrm{Cp}\end{array}$ & $\begin{array}{l}\mathrm{Zr}, \mathrm{Si} \\
\mathrm{Ca}, \mathrm{Mg}, \mathrm{Sl} ; \mathrm{Fo}\end{array}$ & 971 & \\
\hline 18 & $865 \mathrm{Cp}$ & $\mathrm{Ca}, \mathrm{Fo}, \mathrm{Sl} ; \mathrm{Na}, \mathrm{Cr}, \mathrm{Ni}$ & 895 & \\
\hline 19 & $\begin{array}{l}862 \mathrm{Cp} \\
862 \mathrm{NZ}\end{array}$ & $\begin{array}{l}\mathrm{Ca}, \mathrm{Na}, \mathrm{Fo}, \mathrm{Si} ; \mathrm{Mg}, \mathrm{NI}, \mathrm{Cr} \\
\mathrm{Na}, \mathrm{Zr}, \mathrm{Si}\end{array}$ & $>883$ & \\
\hline 20 & $1081 \mathrm{ZS}$ & $\mathrm{Zr}, \mathrm{Si}$ & 7 & $1012-1115$ \\
\hline 21 & $904 \mathrm{Op}$ & $\mathrm{Mg}, \mathrm{SI}$ & & 834.951 \\
\hline 22 & 989 OV & Mg,NI,SI & 994 & \\
\hline 23 & $858 \mathrm{CS}$ & $\mathrm{Ca}, \mathrm{SI}$ & & $807: 1038$ \\
\hline 24 & 8714 (None) & & 800 & \\
\hline 25 & 899 Op & $\mathrm{F}_{0, \mathrm{Si}, \mathrm{Ni}}$ & $>951$ & \\
\hline 26 & 1049 ZS & & & $1012-1115$ \\
\hline 27 & $\leq 642$ (None) & & & $642-1018$ \\
\hline 28 & $<642$ (None) & & & $642-872$ \\
\hline 29 & $1.108 \mathrm{ZS}$ & $\mathrm{Zr}, \mathrm{SI}$ & & $1012 \cdot 1115$ \\
\hline 30 & $1070 \mathrm{Hm}$ & & 1090 & \\
\hline 31 & $939 \mathrm{SO}$ & SI & & $927-1038$ \\
\hline 32 & $1054 \mathrm{NZ}$ & $\mathrm{Na}, \mathrm{Zr}, \mathrm{SI}$ & & $1008-1114$ \\
\hline
\end{tabular}




\begin{tabular}{|c|c|c|c|c|}
\hline $\begin{array}{c}\text { CVS } \\
n\end{array}$ & $\begin{array}{l}\text { Exporimental } \\
\text { TI phase }\end{array}$ & $\begin{array}{l}\text { SEMVDS } \\
\text { elements }\end{array}$ & TI Spinal & $\begin{array}{l}\text { no spinel } \\
T \text { range }\end{array}$ \\
\hline 33 & $\begin{array}{l}967 \mathrm{~Np} \\
967 \mathrm{OV}\end{array}$ & $\begin{array}{l}\mathrm{Na}, \mathrm{Al}, \mathrm{Si} \\
\mathrm{Mg}, \mathrm{NI}, \mathrm{Sl}\end{array}$ & $>1038$ & \\
\hline $34^{\circ}$ & $736 \mathrm{~Np}$ & $\mathrm{Na}, \mathrm{Al}, \mathrm{SI}$ & & 701.872 \\
\hline 35 & $941 \mathrm{~Np}$ & $\mathrm{Na}, \mathrm{Al}, \mathrm{Si}$ & & $895-1115$ \\
\hline 36 & $877 \mathrm{Op}$ & $\mathrm{Fo}_{0, \mathrm{SI}}$ & & 868.1038 \\
\hline 37 & $803 \mathrm{~Np}$ & $\mathrm{Na}, \mathrm{Al}, \mathrm{SI}$ & & $752-1038$ \\
\hline 38 & 1042 Ov & $\mathrm{Mg}_{\mathrm{Si}} \mathrm{Fi}_{\mathrm{O}}$ & $>1115$ & \\
\hline 39 & 1029 ZS & & & $895-1115$ \\
\hline 40 & $862 \mathrm{Cp}$ & $\mathrm{Ca}, \mathrm{Mg}, \mathrm{Si} ; \mathrm{Cr}$ & & 826.928 \\
\hline 41 & $887 \mathrm{Cp}$ & $\mathrm{Ca}_{1} \mathrm{Mg}, \mathrm{Si}_{i} \mathrm{Fo}_{\boldsymbol{\theta}} \mathrm{Ni}, \mathrm{Cr}$ & & 854.936 \\
\hline 42 & $869 \mathrm{Cp}$ & $\mathrm{Ca}, \mathrm{F}_{0}, \mathrm{Mg}, \mathrm{SI}$ & 1093 & \\
\hline 43 & $1090 \mathrm{ZS}$ & & & $1012-1115$ \\
\hline 44 & $884 \mathrm{Cp}$ & $\mathrm{Ca}_{2} \mathrm{Mg}, \mathrm{Si} ; \mathrm{Fo}, \mathrm{Ni}, \mathrm{Cr}$ & & $807-1038$ \\
\hline 45 & $884 . \mathrm{Cp}$ & $\mathrm{Ca}_{1} \mathrm{Mg}, \mathrm{Sl}_{;} \mathrm{Fo}_{\mathrm{\theta}} \mathrm{NI}, \mathrm{Cr}$ & $>956$ & \\
\hline 46 & $858 \mathrm{Cp}$ & $\mathrm{Ca}, \mathrm{Cr}, \mathrm{Fo}_{0}, \mathrm{Mg}_{2} \mathrm{NI}, \mathrm{Si}$ & 1004 & \\
\hline 47 & $877 \mathrm{Cp}$ & $\mathrm{Ca}, \mathrm{Mg}, \mathrm{Sl} ; \mathrm{Na}, \mathrm{Fe}, \mathrm{Ni}, \mathrm{Cr}$ & & $807-1038$ \\
\hline 48 & $868 \mathrm{Cp}$ & $\mathrm{Ca}, \mathrm{Mg}, \mathrm{Si}_{i} \mathrm{Ni}, \mathrm{Fo}_{\mathbf{\theta}} \mathrm{Cr}$ & & $807-1038$ \\
\hline 49 & $847 \mathrm{Cp}$ & $\mathrm{Ca}_{2} \mathrm{Mg}_{2} \mathrm{Fo}, \mathrm{Sl} ; \mathrm{NI}, \mathrm{Cr}$ & 957 & \\
\hline 60 & $\begin{array}{l}922 \mathrm{ZS} \\
922 \mathrm{Cp}\end{array}$ & & 1025 & \\
\hline 51 & $\begin{array}{l}856 \mathrm{Cp} \\
856 \mathrm{NZ} \\
\end{array}$ & $\begin{array}{l}\mathrm{Ca}, \mathrm{Na}, \mathrm{Fo}_{\theta}, \mathrm{Sl} ; \mathrm{Mg}, \mathrm{Cr}, \mathrm{Ni} \\
\mathrm{Na}, \mathrm{Zr}, \mathrm{SI}\end{array}$ & 899 & \\
\hline 52 & $9730 p$ & $\mathrm{Fo}_{0, \mathrm{Cr}, \mathrm{SI}}$ & $>1038$ & \\
\hline 53 & \multicolumn{4}{|c|}{ Radioactive glass $\cdots$ not measured } \\
\hline 54 & \multicolumn{4}{|c|}{ Radioactive glass - not measured } \\
\hline 55 & $\begin{array}{l}944 \mathrm{CO} \\
906 \mathrm{Cp}\end{array}$ & $\mathrm{Na}, \mathrm{Ca}, \mathrm{Fo}_{\mathbf{b}}, \mathrm{Cr}, \mathrm{SI}$ & $>1118$ & \\
\hline 56 & $\begin{array}{l}880 \mathrm{Cp} \\
880 \mathrm{NZ}\end{array}$ & $\begin{array}{l}\mathrm{Na}, \mathrm{Ca}, \mathrm{F}_{\theta}, \mathrm{Cr}, \mathrm{Si} \\
\mathrm{Na}, \mathrm{Zr}, \mathrm{Si}\end{array}$ & 1117. & \\
\hline 57 & $\begin{array}{c}1036 \mathrm{CO} \\
945 \mathrm{Cp} \\
\end{array}$ & $\begin{array}{l}\mathrm{Cr}_{1} \mathrm{Fe}_{\boldsymbol{\theta}} \\
\mathrm{Na}, \mathrm{Ca}, \mathrm{F}_{\boldsymbol{\theta}}, \mathrm{Cr}, \mathrm{Sl}\end{array}$ & 953 & \\
\hline 58 & $\begin{array}{l}906 \mathrm{OV} \\
902 \mathrm{Mg2B2}\end{array}$ & $\begin{array}{l}M g, S i ; N I \\
s(?) \mathrm{Mg}\end{array}$ & (?) & \\
\hline 59 & 71550 & Si & & $699 \cdot 1038$ \\
\hline
\end{tabular}

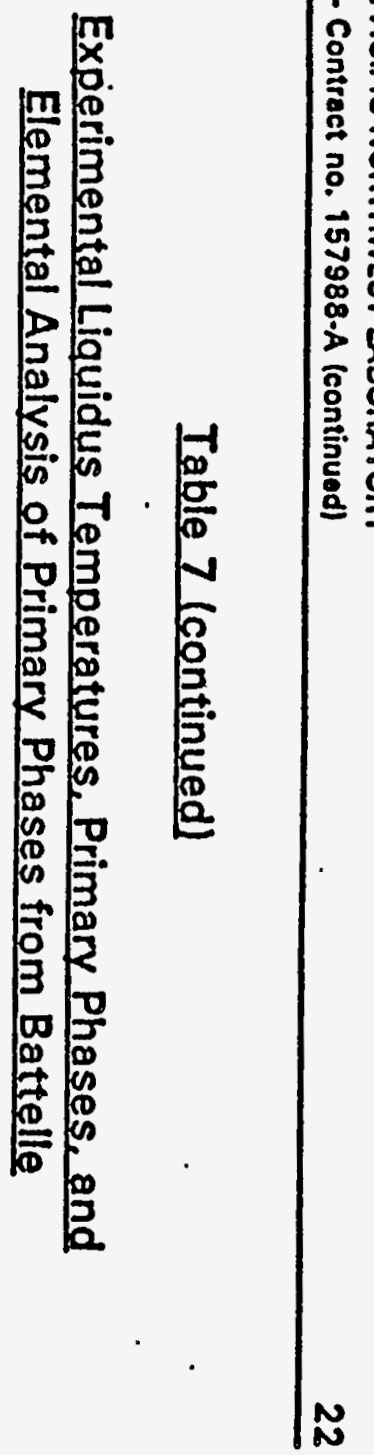




\begin{tabular}{|c|c|c|c|c|}
\hline CVS & $\begin{array}{c}\text { Experimenlal } \\
\text { TI phase }\end{array}$ & $\begin{array}{l}\text { SEMEDS } \\
\text { olements }\end{array}$ & TI Spinol & $\begin{array}{c}\text { no spinel } \\
\text { T range } \\
\end{array}$ \\
\hline 60 & $788 \mathrm{SO}$ & SI & & $752-966$ \\
\hline 61 & 792 SO & Si & & $717-793$ \\
\hline 62 & $<554$ (None) & & & $554-1028$ \\
\hline 63 & $927 \mathrm{~Np}$ & $\mathrm{Na}, \mathrm{Al}, \mathrm{SI}$ & & $868-1038$ \\
\hline 64 & $8950 p$ & $\mathrm{~F}_{\theta, \mathrm{Sl}} \mathrm{CO}_{\mathrm{r}, \mathrm{NI}}$ & 983 & \\
\hline 65 & $21114 \mathrm{ZS}$ & & & $1066-19.14$ \\
\hline 66 & $799 \mathrm{~Np}$ & $\mathrm{Na}, \mathrm{Al}, \mathrm{SI}$ & $>931$ & \\
\hline 67 & $1022 \mathrm{CO}$ & $\mathrm{Cr}$ & & 952.1066 \\
\hline 68 & $>1114 \mathrm{CO}$ & $\mathrm{Cr}$ & & $755-874$ \\
\hline 69 & $\begin{array}{r}1114 \mathrm{CO} \\
983 \mathrm{Op}(?) \\
\end{array}$ & $\begin{array}{l}\mathrm{Cr} \\
\mathrm{Cr}_{1} \mathrm{Fo}_{\mathrm{o}, \mathrm{SI}}\end{array}$ & $>1114$ & \\
\hline 70 & $\begin{array}{c}1114 \mathrm{CO} \\
818 \mathrm{LA} \\
\end{array}$ & $\mathrm{Al}, \mathrm{SI}$ & & 642.931 \\
\hline 71 & $8900 p$ & $F_{\theta, C r, S I}$ & 1004 & \\
\hline 72 & $794 \mathrm{~Np}$ & & $>868$ & \\
\hline 73 & $816 \mathrm{LA}$ & $\mathrm{Al}, \mathrm{SI}$ & & 793.874 \\
\hline 74 & $850 \mathrm{Cp}$ & $\mathrm{Ca}, \mathrm{Fo}_{0} \mathrm{Sl}_{i} \mathrm{NI}$ & 1066 & \\
\hline 75 & $816 \mathrm{LA}$ & Al,SI & & $807-1038$ \\
\hline 76 & $\begin{array}{l}761 \mathrm{Cp} \\
744 \mathrm{LS} \\
\end{array}$ & $\begin{array}{l}\mathrm{Ca}, \mathrm{Fo}_{0}, \mathrm{Sl} ; \mathrm{Na} \\
\mathrm{SI}\end{array}$ & & $712-924$ \\
\hline 77 & $1027 \mathrm{NZ}$ & $\mathrm{Na}, \mathrm{Zr}, \mathrm{SI}$ & & $964 \cdot 1154$ \\
\hline 78 & 112925 & & & 1032.1154 \\
\hline 79 & $949 \mathrm{SO}$ & SI & 31154 & \\
\hline 80 & $>115425$ & $\mathrm{Zr}, \mathrm{Si}$ & 1129 & \\
\hline 81 & $>1154(2)$ & Si & 1013 & \\
\hline 82 & 745 LS & $\mathrm{Si}$ & & 693.924 \\
\hline 83 & $\begin{array}{l}741 \mathrm{CS} \\
741 \mathrm{LS} \\
\end{array}$ & $\begin{array}{l}\mathrm{Ca}, \mathrm{Si} \\
\mathrm{Si} \\
\end{array}$ & & 719.924 \\
\hline 84 & $966 \mathrm{NZ}$ & $\mathrm{Na}, \mathrm{Zr}, \mathrm{SI}$ & & $869-1028$ \\
\hline 85 & $>1150(?)$ & Si & & $1042-1154$ \\
\hline 86 & $1015 \mathrm{~Np}$ & $\mathrm{Na}, \mathrm{Al}, \mathrm{Ca}, \mathrm{Sl} ; \mathrm{S}, \mathrm{Cr}$ & & 067.1154 \\
\hline 87 & $<571$ (none) & & & $571-874$ \\
\hline 88 & 92920 & & & $801-1152$ \\
\hline 89 & $838 \mathrm{Cp}$ & $\mathrm{Ca}, \mathrm{Fo}_{0} \mathrm{Si}_{j} \mathrm{Mg}$ & & $752-924$ \\
\hline
\end{tabular}

\begin{tabular}{|c|c|c|c|c|}
\hline$\overline{\mathrm{CVS}}$ & $\begin{array}{c}\text { Experimenlal } \\
\text { TI phase }\end{array}$ & $\begin{array}{l}\text { SEMIEDS } \\
\text { olements }\end{array}$ & TI Spinel & $\begin{array}{c}\text { no spinel } \\
\text { T range }\end{array}$ \\
\hline 90 & $897 \mathrm{Op}$ & $\mathrm{F}_{\theta}, \mathrm{SI}_{\mathrm{i}} \mathrm{Cr}$ & & 841.967 \\
\hline 91 & $886 \mathrm{SO}$ & $\mathrm{Si}$ & $>967$ & \\
\hline 92 & $897 \mathrm{ZS}$ & & & \\
\hline 93 & $782 \mathrm{Cp}$ & $\mathrm{Na}, \mathrm{Ca}, \mathrm{F}_{\theta}, \mathrm{Si}$ & $>983$ & \\
\hline 94 & $663 \mathrm{CS}$ & $\mathrm{CaSi}$ & & $642-983$ \\
\hline 95 & $804 \mathrm{Cp}$ & $\mathrm{Ca}, \mathrm{Fo}_{0} \mathrm{Sl} ; \mathrm{Na}, \mathrm{Mg}, \mathrm{Tl}$ & & 712.924 \\
\hline 96 & $\begin{array}{l}946 \mathrm{ZS} \\
946 \mathrm{Cp}\end{array}$ & $\mathrm{Ca}_{1} \mathrm{Mg}_{2} \mathrm{Sl}_{;} \mathrm{F}_{\theta}$ & 1010 & \\
\hline 97 & $\begin{array}{l}858 \mathrm{Cp} \\
858 \mathrm{NZ} \\
\end{array}$ & $\mathrm{Ca}_{0} \mathrm{Na}, \mathrm{Fo}_{\theta} \mathrm{Sl}_{;} \mathrm{Cr}, \mathrm{Ni}$ & 887 & \\
\hline 98 & $740 \mathrm{~Np}$ & & & 717.874 \\
\hline 89 & $847 \mathrm{Cp}$ & $\mathrm{Ca}, \mathrm{Fo}_{\mathrm{S}} \mathrm{Si} \mathrm{Na}$ & 888 & \\
\hline 100 & $824 O p$ & $F_{0, S I}$ & 1030 & \\
\hline
\end{tabular}




\section{Table 8}

Calculated Liquidus Temperatures and Primary Phases for CVS Glasses

\begin{tabular}{|c|c|c|c|c|c|}
\hline $\begin{array}{l}\text { ID } \\
\quad \\
\end{array}$ & $\begin{array}{c}\text { CVS } \\
\quad\end{array}$ & $\begin{array}{l}\text { Calc } \\
\pi\end{array}$ & $\begin{array}{l}\text { ulated } \\
\text { Phase }\end{array}$ & $\underset{\text { spinel }}{n}$ & Comments \\
\hline $\mathfrak{\imath}$ & 1 & $\begin{array}{l}907 \\
850 \\
\end{array}$ & $\begin{array}{l}2 S \\
C p\end{array}$ & 968 & \\
\hline 2 & 2 & .920 & CAS & & Phase contains $\mathrm{Ca}, \mathrm{Si}, \mathrm{Al}$ as observed \\
\hline 3 & 3 & 970 & $\mathrm{Al}_{2} \mathrm{O}_{3}$ & & $X$ (poor agreement) \\
\hline 4. & 4 & 985 & so & & \\
\hline 5 & 5 & 920 & Cp & 9062 & Experimental " $\mathrm{FeSiO}_{3}{ }^{\infty}$ is an unstable compound \\
\hline 6 & 6 & 1116 & ZS & & \\
\hline 7 & 7 & 1155 & 25 & & $\dot{-}$ \\
\hline 8 & 8 & 1201 & ZS & & \\
\hline 9 & 9 & 943 & $\mathrm{Mg}_{2} \mathrm{BO}_{3}$ & 1273 & Experimental marked "?" \\
\hline .10 & 10 & $=$ & $=$ & 997 & \\
\hline 11 & 11 & 929 & CAS & 1111 & Phase contains $\mathrm{Ca}$, Si as observed \\
\hline 12 & 12 & 831 & NCS2 & 946 & Experimental " $\mathrm{FeSiO}_{3}$ " is an unstable ompound \\
\hline 13 & 13 & $\ldots$ & - & & \\
\hline 14 & 14 & $\ldots$ & 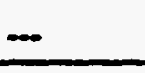 & & \\
\hline 15 & 15 & $\begin{array}{l}859 \\
873 \\
\end{array}$ & $\begin{array}{l}\text { Ov } \\
\text { LS }\end{array}$ & & $\mathrm{Ov}=\mathrm{Mg}_{2} \mathrm{SiO}_{4}$ \\
\hline 16 & 16 & 1153 & $\mathrm{~Np}$ & & $X$ (poor agreement) - See Note (a) \\
\hline 17 & 17 & $\begin{array}{l}907 \\
888 \\
\end{array}$ & $\begin{array}{l}\text { NCSI } \\
\mathrm{Cp}_{p} \\
\end{array}$ & 1108 & Phase contains $\mathrm{Ca}$, Si as observed \\
\hline 18 & 18 & $\begin{array}{l}1216 \\
1113 \\
\end{array}$ & $\begin{array}{l}\text { Ov } \\
20\end{array}$ & & $\begin{array}{l}X \text { (poor agreement) } \\
\text { OK }\end{array}$ \\
\hline 19 & 19 & $\begin{array}{l}907 \\
850 \\
\end{array}$ & $\begin{array}{l}Z S \\
\mathrm{Cp}\end{array}$ & & \\
\hline 20 & 20 & $\begin{array}{l}907 \\
850 \\
\end{array}$ & $\begin{array}{l}Z S \\
C_{p}\end{array}$ & & \\
\hline 21 & 21 & 985 & so & & \\
\hline 22 & 22 & 1201 & Zs & & \\
\hline
\end{tabular}


BATTELLE - PACIFIC NORTHWEST LABORATORY

Final Roport - Contract no. 157988-A (continued)

\begin{tabular}{|c|c|c|c|c|c|}
\hline ID & CVS & $\begin{array}{l}\text { Calc } \\
\pi\end{array}$ & $\begin{array}{l}\text { sulated } \\
\text { Phase }\end{array}$ & $\underset{\text { spinel }}{\pi}$ & Comments \\
\hline 23 & 23 & 890 & $C_{p}-$ & 993 & \\
\hline 24 & 1 & 834 & 25 & 1065 & $\begin{array}{l}\mathrm{X} \text { (poor agreement) - possible inprovement if } \mathrm{Ni} \\
\text { included in Ov model }\end{array}$ \\
\hline 25 & 2 & $\begin{array}{l}871 \\
870\end{array}$ & $\begin{array}{l}\mathrm{Hm} \\
\mathrm{Cp}\end{array}$ & 1152 & , \\
\hline 26 & 3 & $\begin{array}{l}894 \\
835\end{array}$ & $\begin{array}{l}2 S \\
C_{p}\end{array}$ & & - \\
\hline 27 & 4 & 801 & $C_{p}$ & & \\
\hline 28 & 5 & 814 & Albite & 982 & $\begin{array}{l}\text { Chemical analysis questionable. Small crystals } \\
\text { and interference from glass }\end{array}$ \\
\hline 29 & 6 & 898 & $C_{p}$ & & \\
\hline 30 & 7 & 770 & $N p$ & 1116 & $\begin{array}{l}X \text { (poor agreement) - possible improvement if } \mathrm{Ni} \\
\text { included in Ov model }\end{array}$ \\
\hline 31 & 8 & 876 & $C_{p}$ & & \\
\hline 32 & 9 & 1038 & ZS & & \\
\hline 33 & 10 & $\infty$ & $\infty$ & 866 & \\
\hline 34 & 11 & 1005 & ZS & & \\
\hline 35 & 12 & 817 & $C_{p}$ & & \\
\hline 36 & 13 & 872 & $C_{p}$ & & \\
\hline 37 & 14 & 1062 & 25 & & \\
\hline 38 & 15 & $\infty$ & $\cdots$ & 1001 & \\
\hline 39 & 16 & $\begin{array}{l}889 \\
851\end{array}$ & $\begin{array}{l}N Z \\
C_{p}\end{array}$ & 885 & \\
\hline 40 & 17 & $\begin{array}{l}907 \\
850\end{array}$ & $\begin{array}{l}2 S \\
C p\end{array}$ & & \\
\hline 41 & 18 & $\begin{array}{l}889 \\
851\end{array}$ & $\begin{array}{l}N Z \\
C p\end{array}$ & & \\
\hline 42 & 19 & $\begin{array}{l}890 \\
853\end{array}$ & $\begin{array}{l}N Z \\
C p\end{array}$ & & \\
\hline 43 & 20 & 1081 & Zs & & \\
\hline 44 & 21 & 880 & so & & $X$ (poor agreement) \\
\hline 45 & 22 & 1004 & Ov & 915 & \\
\hline 46 & 23 & 859 & NCS1 & & Phase contains $\mathrm{Ca}_{2}$ Si as observed \\
\hline
\end{tabular}


BATTELLE - PACIFIC NORTHWEST LABORATORY

Final Report - Contract no. 157988-A (continued)

\begin{tabular}{|c|c|c|c|c|}
\hline $\begin{array}{l}10 \\
\vdots\end{array}$ & $\begin{array}{c}\text { CVS } \\
\end{array}$ & $\begin{array}{l}\text { Calculated } \\
\pi \text { Phase }\end{array}$ & $\begin{array}{c}\pi \\
\text { spinel }\end{array}$ & Comments \\
\hline 47 & 24 & $\infty \quad \infty$ & 1019 & . \\
\hline 48 & 25 & $900 \mathrm{Cp}$ & & \\
\hline 49 & 26 & $1039 \mathrm{ZS}$ & & \\
\hline 50 & 27 & $\infty \quad \infty$ & & \\
\hline 51 & 28 & $\infty \quad \infty$ & & \\
\hline 52 & 29 & 107725 & & \\
\hline 53 & 30 & $898 \mathrm{~Np}$ & 913 & $x$ (poor agreement) \\
\hline 54 & 31 & 897 so & & . \\
\hline 55 & 32 & $1055 \mathrm{NZ}$ & & \\
\hline 56 & 33 & $969 \mathrm{~Np}$ & 753 & \\
\hline 57 & 34 & $1103 \mathrm{~Np}$ & & $X$ (poor agreement) - See Note (a) \\
\hline 58 & 35 & $971 \mathrm{~Np}$ & 914 & \\
\hline 59 & 36 & $909 C_{p}$ & & $\cdot$ \\
\hline 60 & 37 & $810 \mathrm{~Np}$ & & \\
\hline 69 & 38 & $954 \mathrm{~Np}$ & & $X$ (poor agreement) - See Note (a) \\
\hline 62 & 39 & $996 \quad N Z$ & & \\
\hline 63 & 40 & $853 \mathrm{Cp}$ & & . \\
\hline 64 & 41 & $907 C_{p}$ & & \\
\hline 65 & 42 & $967 C_{p}$ & 1018 & \\
\hline 66 & 43 & $1057 \mathrm{ZS}$ & & \\
\hline 67 & 44 & $\begin{array}{ll}895 & \mathrm{zS} \\
865 & \mathrm{Cp} \\
\end{array}$ & & See Note (b) \\
\hline 68 & 45 & $\begin{array}{ll}945 & Z S \\
865 & \mathrm{Cp} \\
\end{array}$ & & See Note (b) \\
\hline 69 & 46 & $\begin{array}{ll}866 & 2 S \\
815 & C p \\
\end{array}$ & 1051 & See Note (b) \\
\hline 70 & 47 & $\begin{array}{ll}914 & N Z \\
840 & C_{p} \\
\end{array}$ & & See Note $(b)$ \\
\hline 79 & 48 & $\begin{array}{ll}798 & 2 S \\
781 & C_{p}\end{array}$ & & $\begin{array}{l}X \text { (poor agreement) - problem could be related to } \\
\text { high } \mathrm{Cr} \text { and } \mathrm{Ni} \text { contents of solid phases (not } \\
\text { modeled) }\end{array}$ \\
\hline
\end{tabular}


BATTELLE - PACIFIC NORTHWEST LABORATORY.

Final Report - Contract no. 157988-A (continued)

\begin{tabular}{|c|c|c|c|c|c|}
\hline ID & $\begin{array}{c}\text { CVS } \\
\text {. }\end{array}$ & $\begin{array}{l}\text { Calc } \\
\mathbf{n}\end{array}$ & $\begin{array}{c}\text { eulated } \\
\text { Phase }\end{array}$ & $\underset{\text { spinel }}{\pi}$ & Comments \\
\hline 72 & 49 & $\begin{array}{l}818 \\
815\end{array}$ & $\begin{array}{l}2 s \\
\text { Cp }\end{array}$ & 930 & See Note (b) \\
\hline 73 & 50 & $\begin{array}{l}907 \\
850\end{array}$ & $\begin{array}{l}2 S \\
C_{p}\end{array}$ & & • \\
\hline 74 & 51 & $\begin{array}{l}890 \\
884 \\
853\end{array}$ & $\begin{array}{l}N Z \\
Z S \\
C p\end{array}$ & 889 & \\
\hline 75 & 52 & 973 & Zs & & $?$ (good $T$ agreement) \\
\hline 76 & 53 & $\infty$ & $\infty$ & & \\
\hline 77 & 54 & $-\infty$ & $\infty$ & & . \\
\hline 78 & 55 & 1028 & CO(R) & 1124 & $\begin{array}{l}\text { CO(R) temperature will be lower if a significant } \\
\text { amount of spinel precipitates first }\end{array}$ \\
\hline 79 & 56 & $\begin{array}{l}963 \\
884\end{array}$ & $\begin{array}{l}\text { CO(R) } \\
\text { ZS }\end{array}$ & 1124 & $\begin{array}{l}\text { If } C O(R) \text { precipitates in ID\# } 78,80 \text { then it must } \\
\text { also precipitate here }\end{array}$ \\
\hline 80 & 57 & $\begin{array}{l}995 \\
853\end{array}$ & $\begin{array}{l}\text { CO(R) } \\
C_{p}\end{array}$ & 735 & . \\
\hline 81 & 58 & 944 & LA & & $X$ poor agreement as regards phase \\
\hline 82 & 59 & $\begin{array}{l}885 \\
768\end{array}$ & $\begin{array}{l}N p \\
C p\end{array}$ & & $X$ (poor agreement) - See Note (a) \\
\hline 83 & 60 & $\begin{array}{l}784 \\
777\end{array}$ & $\begin{array}{l}N Z \\
C_{p}\end{array}$ & & $\begin{array}{l}\text { Experimental observation of SO probably related } \\
\text { to formation of two-phase liquid }\end{array}$ \\
\hline 84 & 61 & 782 & $C_{p}$ & & $\begin{array}{l}\text { Experimental observation of SO probably related } \\
\text { to formation of two-phase liquid }\end{array}$ \\
\hline 85 & 62 & $\infty$ & $\infty$ & & \\
\hline 86 & 63 & 1215 & Np & & $X$ (poor agreement) - See Note (a) \\
\hline 87 & 64 & $\begin{array}{l}921 \\
907\end{array}$ & $\begin{array}{l}N 2 \\
C_{p}\end{array}$ & 957 & See Note (b) \\
\hline 88 & 65 & 1122 & ZS & & $\cdot$ \\
\hline 89 & 66 & 872 & Np & & \\
\hline 90 & 67 & 1025 & CO(R) & & \\
\hline .91 & 68 & 1732 & CO(R) & & \\
\hline 92 & 69 & 1663 & CO(R). & & \\
\hline 93 & 70 & 1481 & CO(R) & & \\
\hline
\end{tabular}


BATTELLE - PACIFIC NORTHWEST LABORATORY

Final Report - Contract no. 157988-A (continued)

\begin{tabular}{|c|c|c|c|c|}
\hline $\begin{array}{l}10 \\
+ \\
\end{array}$ & $\begin{array}{c}\text { CVS } \\
*\end{array}$ & $\begin{array}{l}\text { Calculated } \\
\text { I Phase }\end{array}$ & $\prod_{\text {spinel }}^{\pi}$ & Comments \\
\hline 94 & 71 & $\begin{array}{ll}921 & N Z \\
907 & C_{p} \\
\end{array}$ & 929 & $?$ (satisfactory agreement in $\pi$ ) \\
\hline 95 & 72 & 872 Np & & \\
\hline 96 & 73 & $\begin{array}{ll}851 & \text { Np } \\
762 & \text { LA } \\
\end{array}$ & & $\dot{-}$ \\
\hline 97 & 74 & $812 \dot{c p}$ & 1123 & \\
\hline 98 & 75 & $\begin{array}{ll}887 & \mathrm{~Np} \\
774 & \mathrm{LA} \\
\end{array}$ & & \\
\hline 99 & 76 & $892 C_{p}$ & & $X$ poor agreement in $T$ (correct phase) \\
\hline 100 & 77 & $979 \mathrm{NZ}$ & & \\
\hline 101 & 78 & $1074 \mathrm{NZ}$ & & \\
\hline 102 & 79 & 847 So & & Phase correct. T too low \\
\hline 103 & 80. & 113325 & 1120 & \\
\hline 104 & 81 & $879 \mathrm{Cp}$ & 901 & Experimental marked "?" \\
\hline 105 & 82 & $\begin{array}{ll}796 & N Z \\
734 & \mathrm{Cp} \\
\end{array}$ & & $T$ correct (experimental "LS" was a guess) \\
\hline 106 & 83 & 786 NCS2 & & Phase contains $\mathrm{Ca}, \mathrm{Si}$ as observed \\
\hline 107 & $84^{\circ}$ & $926 \mathrm{NZ}$ & & \\
\hline 108 & 85 & $949 \mathrm{~Np}$ & & Experimental marked "?" \\
\hline 109 & 86 & $1140 \mathrm{~Np}$ & & \\
\hline 110 & 87 & $=-$ & & \\
\hline 191 & 88 & $903 \mathrm{NZ}$ & & \\
\hline 112 & 89 & $\begin{array}{ll}899 & \mathrm{NZ} \\
865 & \mathrm{Cp} \\
\end{array}$ & & See Note (b) \\
\hline 113 & 90 & $815 C_{p}$ & & . \\
\hline 114 & 91 & $\begin{array}{ll}911 & \text { Albite } \\
873 & \text { Np } \\
820 & \text { So } \\
\end{array}$ & 853 & \\
\hline 115 & 92 & $963 \mathrm{ZS}$ & & \\
\hline 116 & 93 & $862 C_{p}$ & 902 & \\
\hline 117 & 94 & $\begin{array}{ll}932 & N p \\
823 & C_{p} \\
\end{array}$ & & $X$ (poor agreement) See Note (a) \\
\hline 118 & 95 & $870 C_{p}$ & & \\
\hline
\end{tabular}




\begin{tabular}{|c|c|c|c|c|c|}
\hline $\begin{array}{l}\text { ID } \\
:\end{array}$ & $\begin{array}{c}\text { CVS } \\
\end{array}$ & $\begin{array}{l}\text { Calk } \\
\pi\end{array}$ & $\begin{array}{c}\text { culated } \\
\text { Phase }\end{array}$ & $\prod_{\text {spinel }}$ & Comments \\
\hline 119 & 96 & $\begin{array}{l}907 \\
850\end{array}$ & $\begin{array}{l}Z S \\
C p\end{array}$ & & \\
\hline 120 & 97 & $\begin{array}{l}890 \\
884 \\
853\end{array}$ & $\begin{array}{l}N Z \\
Z S \\
C p\end{array}$ & & . \\
\hline 121 & 98 & $\begin{array}{r}1903 \\
720\end{array}$ & $\begin{array}{l}\text { Np } \\
\text { Albite }\end{array}$ & - & $X$ (poor agreement) See Note (a) \\
\hline 122 & 99 & 876 & $C_{p}$ & 949 & \\
\hline 123 & 100 & $\begin{array}{l}952 \\
884\end{array}$ & $\begin{array}{l}Z S \\
C p\end{array}$ & 1067 & Experimental " $\mathrm{FeSiO}_{3}{ }^{"}$ is an unstable compound \\
\hline
\end{tabular}

$\mathrm{Cp}_{p}=$ clinopyroxene $=\mathrm{NaFeSi}_{2} \mathrm{O}_{3}-\mathrm{CaMgSi}_{2} \mathrm{O}_{6}$ solid solution

$\mathrm{Hm}=$ hematite $\mathrm{Np}=$ Nepheline $\mathrm{OV}=$ Olivine

$\mathrm{LA}=\mathrm{Li}$-Al silicate $\mathrm{LS}=\mathrm{Li}_{2} \mathrm{SiO}_{3} \quad \mathrm{NZ}=\mathrm{Na} \cdot \mathrm{Zr}$ silicate

$\mathrm{SO}=\mathrm{SiO}_{2} \quad \mathrm{ZO}=\mathrm{ZrO}_{2} \quad \mathrm{ZS}=\mathrm{ZrSiO}_{4}$

$\mathrm{CO}(\mathrm{R})=\mathrm{Cr}_{2} \mathrm{O}_{2}-\mathrm{Fe}_{2} \mathrm{Oi}_{2} \mathrm{Al}_{2} \mathrm{O}_{3}$ (corundum) solid solution

CAS $=\mathrm{CaAl}_{2} \mathrm{Si}_{2} \mathrm{O}_{3}$ (Anorthite)

$\mathrm{NCS1}=\mathrm{Na}_{2} \mathrm{Ca}_{2} \mathrm{Si}_{3} \mathrm{O}_{2} \cdot \mathrm{Na}_{4} \mathrm{CaSi}_{2} \mathrm{O}_{3}$ solid solution

$\mathrm{NCS2}=\mathrm{Na}_{2} \mathrm{Ca}_{2} \mathrm{Si}_{2} \mathrm{O}_{10}$

Note (a) In general, the precipitation of nephiline is poorly predicted because the optimization of the $\mathrm{Na}_{2} \mathrm{O}-\mathrm{Al}_{2} \mathrm{O}_{3}-\mathrm{SiO}_{2}$ diagram is poor in this composition range. If nepheline is calculated as a primary phase, be skeptical.

Note (b) Glass contains low $\mathrm{ZrO}_{2}$. Small amount of $\mathrm{ZS}$ or $\mathrm{NZ}$ precipitate could have been missed in experiments. 


\subsection{Discussion}

Of the 17 compositions for which nepheline (Np) is the calculated primary phase, agreement between experimental and calculated liquidus temperatures is acceptable in only 9 cases. This is as expected from the discussion in Section 2.2. When nepheline is calculated as the primary phase, be skeptical.

In Fig. 1 are plotted experimental versus calculated liquidus temperatures for the 80 compositions (excluding the 17 compositions for which nepheline is calculated as the primary phasel for which experimental liquidus temperatures are given in Table 7. The average deviation is $32^{\circ}$. (If the 17 nepheline points are also included, the average is $47^{\circ}$ ) Eight of the eleven points for which experimental values were indicated as " $>T$ " are also shown on Fig. 1. (The other three, $10 \# 67-69$ are off the scale of Fig. 1 but calculations agree with observations.)

In several glasses (ID\# 67, 68, 69, 70, 72, 87, 112), Zr-containing phases (ZS or NZ) were calculated as primary phases. Although these were not observed experimentally, these glasses contain very low $\mathrm{ZrO}_{2}$ contents, and the small amounts of precipitate could have been overlooked.

Orthopyroxene (Op), either as $\mathrm{MgSiO}_{3}$ or a solution of $\mathrm{MgSiO}_{3}$ and $\mathrm{FeSiO}_{3}$, was never obtained as a primary phase in the calculations. Furthermore, its calculated thermodynamic activity was always very low. That is, only large changes in the optimized thermodynamic properties of this phase, well outside the error limits of the present calculations, would cause it to appear. Furthermore, in many cases, the experimental observations (Table 7 ) indicate an Op phase containing only $\mathrm{Fe}$ and $\mathrm{Si}$. However, pure $\mathrm{FeSiO}_{3}$ is an unstable compound. We thus have no explanation for the experimental observation of the Op phase.

In several cases, $\mathrm{CaSiO}_{3}(\mathrm{CS})$ is shown in Table 7 as the primery experimental phase. In the calculations, the thermodynamic activity of this phase was also always low. However, anorthite (CAS $=\mathrm{CaAl}_{2} \mathrm{Si}_{2} \mathrm{O}_{8}$ ) or a sodium-calcium silicate (NCS1 or NCS2) were observed as primary phase in these samples, and these compounds contain the elements $\mathrm{Ca}, \mathrm{Si}$, (Al) observed by SEM/EDS.

Somewhat better agreement might be obtained by incorporating Ni into the models for some of the solid phases, particularly olivine. Unfortunately, there are few data upon which the modeling could be based.

CVS-Il glasses \#55-57 differ only in the content of $\mathrm{Cr}_{2} \mathrm{O}_{3}$ (and $\mathrm{MnO}_{2}$ ). Hence, if corundum $\left(\mathrm{Cr}_{2} \mathrm{O}_{3}\right.$ solution) precipitates in $\# 55$ and 57 , it would also be expected to precipitate in $\# 56$ as calculated, but this apparently was not observed. 


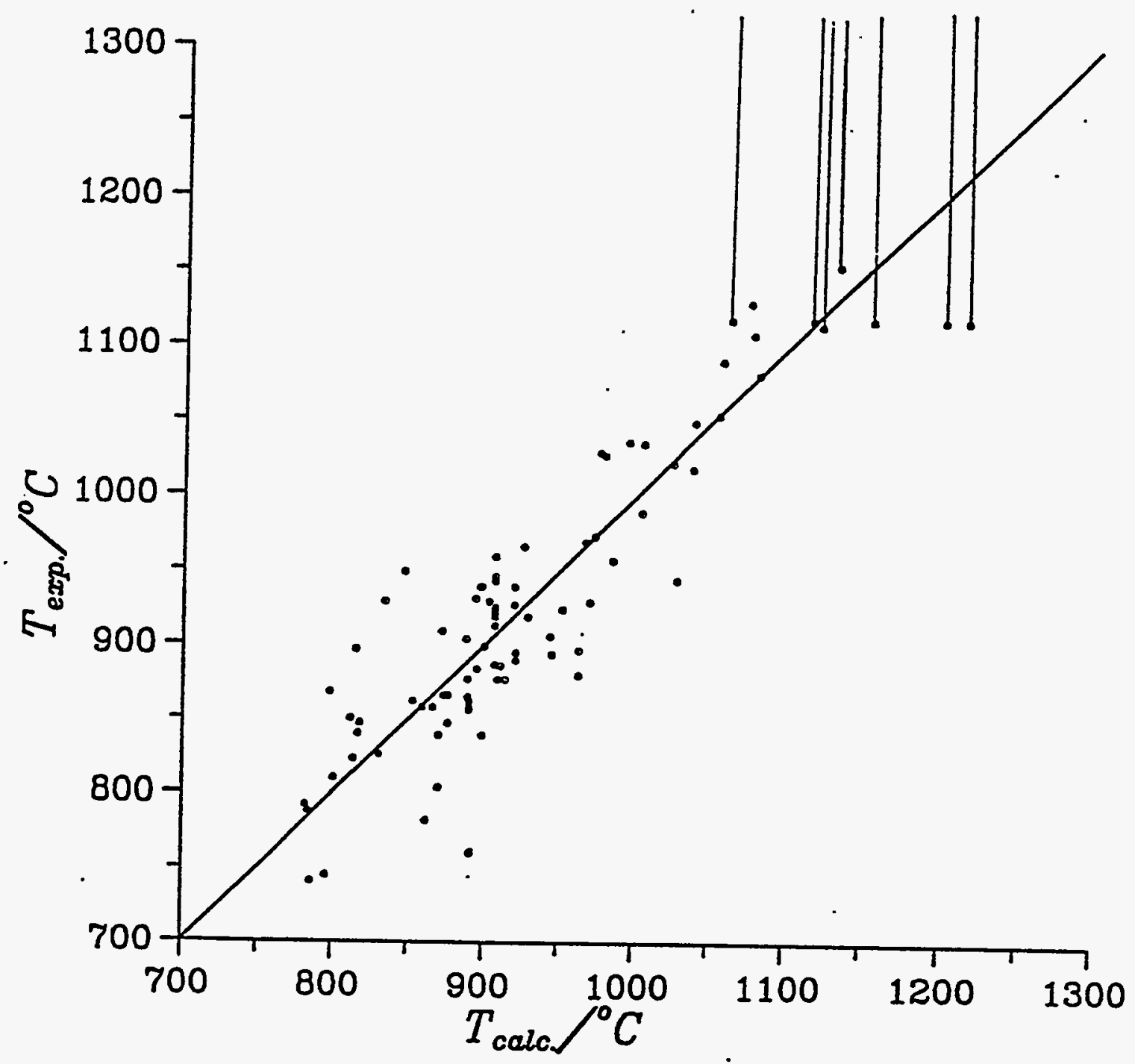

Comparison of Experimental and Calculated Liquidus Temperatures for CVS Glasses (Assuming negligible spinel precipitation)

Fig. 1 


\section{THE SPINEL PHASE}

A two-sublattice model for $(\mathrm{Fe}, \mathrm{Ni})(\mathrm{Fe}, \mathrm{Cr}, \mathrm{Al})_{2} \mathrm{O}_{4}$ spinel solutions has been developed through optimization of available thermodynamic data for the pure components $\left(\mathrm{Fe}_{3} \mathrm{O}_{4}\right.$, $\mathrm{NiCr}_{2} \mathrm{O}_{4}$, etc.) and of available data for equilibria between the spinel phase and other solid phases (corundum, wustite, metal, etc.) as a function of oxygen pressure, temperature and composition in the Fe-Cr-O, Fe-Ni-O, Fe-Al-O, Ni-Cr-O and Fe-Cr-Al-O systems.

Manganese was included in the spinel model by taking literature data for the pure compounds $\mathrm{MnAl}_{2} \mathrm{O}_{4}$ and $\mathrm{Mn}_{3} \mathrm{O}_{4}$, by taking $\Delta \mathrm{H}_{298}^{\circ}$ from the literature for the pure compounds $\mathrm{MnCr}_{2} \mathrm{O}_{4}$ and $\cdot \mathrm{NiMn}_{2} \mathrm{O}_{4}$ and then estimating $\mathrm{S}_{298}^{\circ}$ and $\mathrm{Cp}$ by an increment method, and by using the observed fact that $\mathrm{Mn}_{3} \mathrm{O}_{4}-\mathrm{Fe}_{3} \mathrm{O}_{4}$ solid solutions are nearly ideal.

Good agreement with a large and diverse body of experimental data was obtained. It is thus believed that the thermodynamic properties of the spinel (Fe, $\mathrm{Mn}, \mathrm{Ni}$ )(Fe, $\mathrm{Mn}, \mathrm{Cr}$, $\mathrm{All}_{2} \mathrm{O}_{4}$ phases are well modeled. Inaccuracies in calculations of spinel liquidus temperatures are more likely due to the database for the glass phase, or to the fact that the dissolution of $\mathrm{Mg}$ in the spinel solution was not modeled, or to the experimental results.

The spinel liquidus temperature was calculated for several CVS glasses for which observed spinel liquidus temperatures are reported in Table 7. Results of the calculations at $\cdot P_{\mathrm{O}_{2}}=1$ atm are shown in Table 8. The oxygen pressure had little effect. Decreasing $\mathrm{Po}_{2}$ to $10^{-3} \mathrm{~atm}$ changed the calculated liquidus temperatures only by approximately $3^{\circ} .^{2} \mathrm{~A}$ comparison of Tables 7 and 8 shows that agreement is satisfactory in some cases and poor in others. Out of 24 samples, an average deviation of $68^{\circ}$ was obtained. Of 8 additional samples in which the experimental observations were shown as " $>T$ ", agreement was found in 3.

In the case of samples with ID\# 88 and 89 (CVS-11 \#55, 56), calculation of the spinel liquidus temperature before $\mathrm{Mn}$ was added to the spinel model gave $\mathrm{TI}=809^{\circ}$. That is, adding $\mathrm{Mn}$ to the spinel phase gives a large increase in the calculated temperature and results in much better agreement with the experimental results in these high-Mn(see Table 6) glasses.

\section{SOLUBILITYOF $\mathrm{SO}_{3}, \mathrm{PO}_{2}, \mathrm{Cl}, \mathrm{F}, 1$ AND $\mathrm{H}_{2}$ O IN GLASSES}

Recently we have developed a simple model [93 Pel] which has proved very successful in predicting the solubility of sulfur as sulfide ion, $\mathrm{S}^{2 \cdot}$ (under reducing conditions) in oxide slags and glasses for concentrations up to about $10 \mathrm{wt} . \%$. It is assumed that the $\mathrm{S}^{2-}$ ions substitute nearly ideally for $\mathrm{O}^{2 \cdot}$ ions and for $\mathrm{SiO}_{4}$ groups in the glass structure. Sulfide solubilities can then be predicted from a knowledge only of $\mathrm{G}^{\circ}$ of the pure sulfides $\left(\mathrm{Na}_{2} \mathrm{~S}, \mathrm{CaS}\right.$ etc.) and of the activities of the oxides in the glass (which are given by the quasichemical 
model.) If necessary, constant adjustable parameters, $R T$ In $\gamma_{\text {MS }}$ (where $\dot{y}=$ activity coefficient) may be included. The model has been described in detail [ 93 Pel] and its application to the calculation of sulfide solubilities has been demonstrated.

In the present study, the model has been adapted to permit the calculation of the solubility of other anions $\left(\mathrm{SO}_{4}^{2-}, \mathrm{Cl}, \mathrm{r}, \mathrm{F}, \mathrm{PO}_{4}^{3-}, \mathrm{OH}^{\circ}\right)$ in an oxide slag or glass through substitution for $\mathrm{O}^{2 \cdot}$ ions or $\mathrm{SiO}_{4}$ groups. This has required re-programming of a number of $F * A$ *C"T software modules. written:

Under oxidizing conditions, sulfur dissolves in glass as sulfate. The dissolution can be

$$
\mathrm{O}^{2 \cdot}+\mathrm{SO}_{3}=\mathrm{SO}_{4}^{2 \cdot}
$$

For dilute solutions of $\mathrm{SO}_{4}^{2-}$, the activity of the oxide ion is constant and the activity of $\mathrm{SO}_{4}^{2-}$ abeys Henry's Law. Hence, the weight of dissolved $\mathrm{SO}_{4}$ (or dissolved " $\mathrm{SO}_{3}$ " or dissolved " $\mathrm{S"}$ ) varies directly as $\mathrm{p}_{\mathrm{SO}_{3}}$. Alternatively, the dissolution can be written as:

or as

$$
\begin{aligned}
& \mathrm{O}^{2 \cdot}+\mathrm{SO}_{2}+1 / 2 \mathrm{O}_{2}=\mathrm{SO}_{4}^{2 \cdot} \\
& \mathrm{O}^{2+}+1 / 2 \mathrm{~S}_{2}+3 / 2 \mathrm{O}_{2}=\mathrm{SO}_{4}^{2 *}
\end{aligned}
$$

in which case the weight of dissolved sulfate varies as $\left(\mathrm{P}_{\mathrm{SO}_{2}} \mathrm{P}_{2}^{1 / 2}\right)$ or as $\left(\mathrm{P}_{\mathrm{S}_{2}}^{1 / 2} \mathrm{P}_{\mathrm{O}_{2}}^{3 / 2}\right)$. This behavior has been observed, thereby confirming that under oxidizing conditions the predominant sulfur-containing species in solution is $\mathrm{SO}_{4}^{2+}$.

The dissolution of phosphorus as phosphate under oxidizing conditions has been confirmed by the observation that the weight percent of dissolved phosphorus varies closely as $\left(P_{\mathrm{P}_{2}}^{1 / 2} P_{\mathrm{O}_{2}}^{5 / 4}\right.$ laccording to the mechanism:

$$
3 / 2 \mathrm{O}^{2 \cdot}+1 / 2 \mathrm{P}_{2}+5 / 4 \mathrm{O}_{2}=\mathrm{PO}_{4}^{3}
$$

In the case of $\mathrm{H}_{2} \mathrm{O}$, it has been observed that the weight of dissolved $\mathrm{H}_{2} \mathrm{O}$ varies as $\mathrm{P}_{\mathrm{H}_{2} \mathrm{O}}^{1 / 2}$, thereby indicating dissolution as $\mathrm{OH}^{-}$according to:

$$
1 / 2 \mathrm{O}^{2-}+1 / 2 \mathrm{H}_{2} \mathrm{O}=\mathrm{OH}^{-}
$$

However, there is evidence that some of the hydroxyl groups are bonded to the silicon network. Therefore, the simple model has been modified in this case as discussed below in Section 9.4. 
In the following sections, the model is used to calculate solubilities of sulfate, phosphate, halides and water, and the calculations are compared to the available data. In most cases, no adjustable parameters were required. The model calculations are probably valid to total solute concentrations of about $10 \mathrm{wt} . \%$.

\section{$9.1 \quad \mathrm{SO}_{2}$ Solubilities}

For the case of sulfur solubility as $\mathrm{SO}_{4}^{2 \cdot}$ in glasses, no adjustable model parameters whatsoever were used. The $\mathrm{G}^{\circ}$ values for $\mathrm{Na}_{2} \mathrm{SO}_{4}, \mathrm{Li}_{2} \mathrm{SO}_{4}, \mathrm{CaSO}_{4}$ and $\mathrm{MgSO}_{4}$ were taken directly from the $F^{*} A * C * T$ database. That is, the following comparisons with measured solubilities involved no curve-fitting, and are a priori predictions.

$\mathrm{SO}_{3}$ solubilities in glasses in equilibrium with a gaseous phase have been reported by many authors. Nagashima and Katsura [ $73 \mathrm{Nag}$ ] measured $\mathrm{SO}_{3}$ solubilities in $\mathrm{Na}_{2} \mathrm{O}-\mathrm{SiO}_{2}$ melts at different $\mathrm{SiO}_{2} / \mathrm{Na}_{2} \mathrm{O}$ ratios at different temperatures for constant $\mathrm{P}_{\mathrm{SO}_{2}} \mathrm{PO}_{2}^{1 / 2}=0.08 \mathrm{kPa}$. Their results and calculated values are compared in Table 9 . Similar measurements by Holmquist [66 Holl are compared with calculated values in Table 10. In these experiments, $\mathrm{P}_{\mathrm{SO}_{2}} \mathrm{P}_{\mathrm{O}_{2}}^{1 / 2}$ was also varied and the expected approximately linear relationship between log (wt. $\%$ $\left.\mathrm{SO}_{3}\right)$ and $\log \left(\mathrm{P}_{\mathrm{SO}_{2}} \mathrm{P}_{\mathrm{O}_{2}}^{1 / 2}\right)$ was observed. Papadopoulos [73 Pap] measured $\mathrm{SO}_{3}$ solubilities in predominantly $\mathrm{SiO}_{2}-\mathrm{CaO}-\mathrm{Na}_{2} \mathrm{O}$ glasses at $\mathrm{P}_{\mathrm{SO}_{3}}=0.01 \mathrm{~atm}$. His results (corrected to $P_{\mathrm{sO}_{3}}=0.01 \mathrm{~atm}$ ) are compared to calculated values in Table 11. Fincham and Richardson's [54 Fin] measurements of $\mathrm{SO}_{3}$ solubilities in $\mathrm{CaO}-\mathrm{Al}_{2} \mathrm{O}_{3} \cdot \mathrm{SiO}_{2}$ glasses at $1500^{\circ} \mathrm{C}$ are compared to measurements in Table 12. Other data reported by these authors at $1650^{\circ} \mathrm{C}$ were not sufficiently complete to permit the calculation of $\mathrm{SO}_{3}$ solubilities. However, relative solubilities among glasses of different compositions can be deduced from their results, and these are also compared with calculations in Table 12.

From Tables 9-12 it can be seen that agreement between experimental and calculated solubilities is good to excellent. The best agreement is with the results of Papadopoulos in Table 11. The agreement with the result of Holmquist in Table 10 is not as good at low $\mathrm{SiO}_{2} / \mathrm{Na}_{2} \mathrm{O}$ ratios, but any attempt to improve this agreement by introducing adjustable parameters results in poorer agreement with the other authors. That is, the agreement is believed to be within the error limits of the experiments.

Schreiber et al [87 Sch] measured $\mathrm{SO}_{3}$ solubilities in an alkali borosilicate glass of. composition (wt.\%) 57.9 $\mathrm{SiO}_{2}, 1.0 \mathrm{TiO}_{2}, 0.5 \mathrm{ZrO}_{2}, 14.7 \mathrm{~B}_{2} \mathrm{O}_{3}, 0.5 \mathrm{La}_{2} \mathrm{O}_{3}, 2.0 \mathrm{MgO}, 5.7 \mathrm{Li}_{2} \mathrm{O}$ and 17.7 $\mathrm{Na}_{2} \mathrm{O}$. Results from the authors' smoothed curve are compared with calculated values in Table 13. (In the calculation, $\mathrm{TiO}_{2}$ and $\mathrm{La}_{2} \mathrm{O}_{3}$ were ignored.) Agreement is within the range of scatter of the data points as reported in the article [87 Sch]. 


\section{Table 9}

Calculated $\mathrm{SO}_{3}$ Solubility in $\mathrm{Na}_{2} \mathrm{O} \cdot \mathrm{SiO}_{2}$ Glasses Compared to Measurements of Nagashima and Katsura $173 \mathrm{Nagl}$

$$
\left(P_{\mathrm{SO}_{2}} P_{0_{2}}^{1 / 2}=0.08 \mathrm{kPa}\right)
$$

\begin{tabular}{|c|c|c|c|c|}
\hline \multirow{2}{*}{$\begin{array}{l}\text { Molar Ratio } \\
\left(\mathrm{SiO}_{2} / \mathrm{Na}_{2} \mathrm{O}\right)\end{array}$} & \multirow[t]{2}{*}{$\dot{\mathrm{T}}\left({ }^{\circ} \mathrm{C}\right)$} & \multirow[t]{2}{*}{$1 / T\left(10^{-4} / R\right)$} & \multicolumn{2}{|c|}{ in $W_{\mathrm{sO}_{3}}$ (weight fraction) } \\
\hline & & & expt. & calc \\
\hline 1 & $\begin{array}{l}1300 \\
1250 \\
1100\end{array}$ & $\begin{array}{l}6.36 \\
6.57 \\
7.28 \\
\end{array}$ & $\begin{array}{l}-3.1 \\
-2.8 \\
-0.7\end{array}$ & $\begin{array}{c}-2.7 \\
-2.3 \\
(-2.0)\end{array}$ \\
\hline 2 & $\begin{array}{l}1300 \\
1250 \\
1100 \\
\end{array}$ & $\begin{array}{l}6.36 \\
6.57 \\
7.28 \\
\end{array}$ & $\begin{array}{l}-6.3 \\
-5.8 \\
-2.9\end{array}$ & $\begin{array}{l}-7.4 \\
-6.4 \\
-3.45\end{array}$ \\
\hline 3 & $\begin{array}{l}1300 \\
1250 \\
1100\end{array}$ & $\begin{array}{l}6.36 \\
6.57 \\
7.28\end{array}$ & $\begin{array}{l}-9.7 \\
-9.1 \\
-5.2\end{array}$ & $\begin{array}{l}-9.9 \\
-8.9 \\
.5 .5\end{array}$ \\
\hline
\end{tabular}


Table 10

Calculated $\mathrm{SO}_{3}$ Solubility in $\mathrm{NaO}_{2} \mathrm{O}-\mathrm{SiO}_{2}$ Glasses Compared to Measurements of Holmquist 166 Holl

\begin{tabular}{|c|c|c|c|c|}
\hline \multirow{2}{*}{$T\left({ }^{\circ} \mathrm{C}\right)$} & \multirow{2}{*}{$\begin{array}{l}\left(\mathrm{SiO}_{2} \mathrm{Na}_{2} \mathrm{O}\right) \\
\text { Molar Ratio } \\
\text { - }\end{array}$} & \multirow{2}{*}{$10^{4} \mathrm{P}_{\mathrm{sO}_{2}} \mathrm{P}_{0_{2}}^{1 / 2}$ (atm) } & \multicolumn{2}{|c|}{$\log \left(w t . \% \mathrm{SO}_{3}\right)$} \\
\hline & & & $\begin{array}{c}\text { expt. } \\
\text { (Smoothed) }\end{array}$ & Calc. \\
\hline \multirow[t]{3}{*}{1150} & 2 & $\begin{array}{c}0.3 \\
1 \\
3 \\
\end{array}$ & $\begin{array}{r}-0.32 \\
0.20 \\
0.69 \\
\end{array}$ & $\begin{array}{r}-0.24 \\
0.20 \\
0.52 \\
\end{array}$ \\
\hline & 3 & $\begin{array}{c}3 \\
10 \\
\end{array}$ & $\begin{array}{l}-0.26 \\
(0.71) \\
\end{array}$ & $\begin{array}{r}-0.36 \\
0.10 \\
\end{array}$ \\
\hline & 4 & 10 & -0.22 & -0.40 \\
\hline \multirow[t]{6}{*}{1200} & 1.5 & $\begin{array}{l}1^{\circ} \\
3 \\
\end{array}$ & $\begin{array}{l}0.76 \\
1.06 \\
\end{array}$ & $\begin{array}{l}0.54 \\
0.78 \\
\end{array}$ \\
\hline & 1.75 & $\begin{array}{l}1 \\
3 \\
\end{array}$ & $\begin{array}{l}0.47 \\
0.81 \\
\end{array}$ & $\begin{array}{l}0.15 \\
0.48 \\
\end{array}$ \\
\hline & 2.0 & $\begin{array}{c}1 \\
3 \\
10 \\
\end{array}$ & $\begin{array}{l}0.05 \\
0.46 \\
0.89 \\
\end{array}$ & $\begin{array}{r}-0.24 \\
0.16 \\
0.52 \\
\end{array}$ \\
\hline & 2.5 & $\begin{array}{c}1 \\
3 \\
10 \\
\end{array}$ & $\begin{array}{r}-0.75 \\
-0.22 \\
0.36 \\
\end{array}$ & $\begin{array}{r}-0.85 \\
-0.41 \\
0.05\end{array}$ \\
\hline & 3 & $\begin{array}{c}3 \\
10 \\
30 \\
\end{array}$ & $\begin{array}{r}-0.86 \\
-0.31 \\
0.16 \\
\end{array}$ & $\begin{array}{r}-0.85 \\
-0.34 \\
0.08 \\
\end{array}$ \\
\hline & 4 & $\begin{array}{l}10 \\
30\end{array}$ & $\begin{array}{l}-0.84 \\
-0.25\end{array}$ & $\begin{array}{l}-0.89 \\
-0.41\end{array}$ \\
\hline \multirow[t]{5}{*}{1250} & 9.5 & 1 & 0.48 & 0.20 \\
\hline & 1.75 & 1 & 0.00 & -0.28 \\
\hline & 2 & $\begin{array}{c}1 \\
3 \\
10 \\
30 \\
\end{array}$ & $\begin{array}{l}-0.27 \\
0.02 \\
0.35 \\
0.85 \\
\end{array}$ & $\begin{array}{r}-0.69 \\
-0.24 \\
0.19 \\
0.52\end{array}$ \\
\hline & 2.5 & 10 & -0.15 & -0.37 \\
\hline & 3 & $\begin{array}{l}10 \\
30\end{array}$ & $\begin{array}{l}-0.61 \\
-0.16\end{array}$ & $\begin{array}{l}-0.78 \\
-0.33\end{array}$ \\
\hline
\end{tabular}


Table 11

Calculated $\mathrm{SO}_{3}$ Solubilities in $\mathrm{SiO}_{2}-\mathrm{CaO}-\mathrm{Na}_{2} \mathrm{O}-\left(\mathrm{Al}_{2} \mathrm{O}_{3}-\mathrm{MgO}\right)$ Glasses Compared to Measurements of Papadopoulos 173 Papl

(at $P_{\mathrm{SO}_{3}}=0.01 \mathrm{~atm}$ )

\begin{tabular}{|c|c|c|c|c|c|c|c|}
\hline \multirow{2}{*}{$\mathrm{T}(\mathrm{C})$. } & \multicolumn{5}{|c|}{ Mole fractions } & \multicolumn{2}{|c|}{$\mathrm{SO}_{3}$ (mole fraction) } \\
\hline & $\mathrm{SiO}_{2}$ & $\mathrm{Al}_{2} \mathrm{O}_{3}$ & $\mathrm{CaO}$ & $\mathrm{MgO}$ & $\mathrm{Na}_{2} \mathrm{O}$ & $\begin{array}{c}\text { expt. } \\
\text { (corrected) }\end{array}$ & calc. \\
\hline 1370 & $\begin{array}{l}0.721 \\
0.722 \\
0.741 \\
0.707 \\
0.715 \\
0.710 \\
0.653 \\
0.653 \\
0.657 \\
0.661 \\
0.685 \\
0.778 \\
0.778 \\
\end{array}$ & $\begin{array}{l}0.002 \\
0.001 \\
0.001 \\
0.001 \\
0.001 \\
0.001 \\
0.002 \\
0.002 \\
0.002 \\
0.002 \\
0.002 \\
0.001 \\
0.001 \\
\end{array}$ & $\begin{array}{l}0.136 \\
0.142 \\
0.089 \\
0.200 \\
0.193 \\
0.196 \\
0.195 \\
0.196 \\
0.194 \\
0.195 \\
0.144 \\
0.090 \\
0.144 \\
\end{array}$ & $\begin{array}{l}0.005 \\
0.004 \\
0.003 \\
0.005 \\
0.005 \\
0.005 \\
0.005 \\
0.004 \\
0.005 \\
0.005 \\
0.004 \\
0.004 \\
0.004\end{array}$ & $\begin{array}{l}0.127 \\
0.123 \\
0.159 \\
0.083 \\
0.083 \\
0.085 \\
0.133 \\
0.132 \\
0.131 \\
0.127 \\
0.154 \\
0.123 \\
0.071 \\
\end{array}$ & $\begin{array}{l}0.00745 \\
0.00636 \\
0.00664 \\
0.00280 \\
0.00280 \\
0.00238 \\
0.01216 \\
0.01265 \\
0.01050 \\
0.00967 \\
0.01066 \\
0.00360 \\
0.00133 \\
\end{array}$ & $\begin{array}{l}0.00715 \\
0.00685 \\
0.0103 \\
0.00417 \\
0.00385 \\
0.00420 \\
0.0162 \\
0.0161 \\
0.0151 \\
0.0137 \\
0.0156 \\
0.0044 \\
0.00185\end{array}$ \\
\hline $\begin{array}{l}1373 \\
1397 \\
1403 \\
1427 \\
1433 \\
1451 \\
1453 \\
1483\end{array}$ & $\begin{array}{l}0.653 \\
0.660 \\
0.659 \\
0.677 \\
0.669 \\
0.660 \\
0.665 \\
0.666\end{array}$ & $\begin{array}{l}0.002 \\
0.002 \\
0.002 \\
0.002 \\
0.002 \\
0.002 \\
0.002 \\
0.002\end{array}$ & $\begin{array}{l}0.196 \\
0.198 \\
0.194 \\
0.187 \\
0.192 \\
0.201 \\
0.194 \\
0.199\end{array}$ & $\begin{array}{l}0.004 \\
0.005 \\
0.004 \\
0.005 \\
0.004 \\
0.005 \\
0.006 \\
0.005\end{array}$ & $\begin{array}{l}0.132 \\
0.128 \\
0.131 \\
0.123 \\
0.128 \\
0.128 \\
0.129 \\
0.125\end{array}$ & $\begin{array}{l}0.0126 \\
0.0115 \\
0.0107 \\
0.0066 \\
0.0062 \\
0.0063 \\
0.0050 \\
0.0038\end{array}$ & $\begin{array}{l}0.0157 \\
0.0108 \\
0.0108 \\
0.0060 \\
0.0069 \\
0.0063 \\
0.0058 \\
0.0040\end{array}$ \\
\hline
\end{tabular}




\section{Table 12}

Calculated Sulphate Capacities, $\mathrm{C}_{\mathrm{SO}_{4}}$ of $\mathrm{CaO}-\mathrm{Al}_{2} \mathrm{O}_{3}-\mathrm{SiO}_{2}$ Glasses Compared to Measurements of Fincham and Richardson [54 Fin]

$$
C_{S_{O_{4}}}=\left(W t . \% S / P_{S_{2}}^{1 / 2} P_{O_{2}}^{3 / 2}\right) \text { where } P=\text { atm. }
$$

\begin{tabular}{|c|c|c|c|c|c|}
\hline \multirow{2}{*}{$\begin{array}{c}\text { Slag } \\
\text { Number } \\
\text { [54 Fin] }\end{array}$} & \multicolumn{3}{|c|}{ Composition (wt.\%) } & \multicolumn{2}{|c|}{$\log \mathrm{C}_{50_{4}}$ at $1500^{\circ} \mathrm{C}}$. \\
\hline & $\mathrm{CaO}$ & $\mathbf{A}_{2} \mathrm{O}_{2}$ & $\mathrm{SiO}_{2}$ & expt. & calc \\
\hline \multirow[t]{3}{*}{$\begin{array}{l}\text { S1 } \\
\text { S2 } \\
\text { S4 } \\
\text { S5 } \\
\text { S6 } \\
\end{array}$} & $\begin{array}{l}37 \\
32.5 \\
54 \\
41 \\
45.8 \\
\end{array}$ & $\begin{array}{l}27 \\
26.5 \\
1 \\
52 \\
18\end{array}$ & $\begin{array}{c}36 \\
41 \\
45 \\
7 \\
36.2\end{array}$ & $\begin{array}{l}6.70 \\
6.50 \\
7.18 \\
7.83 \\
7.20\end{array}$ & $\begin{array}{l}6.43 \\
5.94 \\
7.02 \\
7.60 \\
7.05\end{array}$ \\
\hline & & & & \multicolumn{2}{|c|}{$\log C_{2 \mathrm{O}_{4}}-\log \mathrm{C}_{5 \mathrm{O}_{4}}$ (slag 56) at $1650^{\circ} \mathrm{C}$} \\
\hline & & & & expt. & calc. \\
\hline $\begin{array}{l}\text { S11 } \\
\text { S12 } \\
\text { S14 } \\
\text { S15 } \\
\text { S16 }\end{array}$ & $\begin{array}{l}54 \\
48 \\
35.5 \\
41 \\
52.5\end{array}$ & $\begin{array}{l}0 \\
0 \\
64.5 \\
59 \\
47.5\end{array}$ & $\begin{array}{r}46 \\
52 \\
0 \\
0 \\
0\end{array}$ & $\begin{array}{r}-0.25 \\
-0.35 \\
-0.20 \\
0.15 \\
0.95\end{array}$ & $\begin{array}{r}-0.03 \\
-0.46 \\
0.22 \\
0.63 \\
1.33\end{array}$ \\
\hline
\end{tabular}

Table 13

Calculated $\mathrm{SO}_{3}$ Solubility in Alkali Borosilicate Glass at $1150^{\circ} \mathrm{C}$ Compared to Measurements of Schreiber et al [87 Sch]

\begin{tabular}{|c|c|c|}
\hline \multirow{2}{*}{$\log \left(10^{4} P_{\mathrm{sO}_{2}} P_{\mathrm{O}_{2}}^{1 / 2}\right)(\mathrm{atm})$} & \multicolumn{2}{|c|}{$\log (\mathrm{wt} . \% \mathrm{~S})$} \\
\cline { 2 - 3 } & expt. (smoothed) & calc. \\
\hline 1.0 & -0.44 & -0.77 \\
1.5 & 0.03 & -0.32 \\
\hline
\end{tabular}


Table 14

Calculated $\mathrm{SO}_{3}$ Solubilities in Glass in Equilibrium with a Molten Salt Phase for

Glass Compositions used by Sullivan 194 Sull

Glass Compositions (wt. \%)

\begin{tabular}{|c|c|c|c|}
\hline & $W L-3$ & $W L-4$ & $W L-5$ \\
\cline { 2 - 4 } $\mathrm{SiO}_{2}$ & 59.2 & 57.1 & 55.0 \\
$\mathrm{~B}_{2} \mathrm{O}_{3}$ & 11.4 & 10.8 & 10.3 \\
$\mathrm{Na}_{2} \mathrm{O}$ & 11.2 & 11.2 & 11.2 \\
$\mathrm{Li}_{2} \mathrm{O}$ & 6.8 & 6.8 & 6.8 \\
$\mathrm{Al}_{2} \mathrm{O}_{3}$ & 6.2 & 7.6 & 9.1 \\
$\mathrm{P}_{2} \mathrm{O}_{5}$ & 1.1 & 1.4 & 1.6 \\
$\mathrm{SO}_{3}$ & 1.3 & 1.6 & 1.9 \\
Others & 2.8 & 3.5 & 4.1 \\
\hline
\end{tabular}

$\mathrm{SO}_{3}$ Solubilities in Glass in Equilibrium with Liquid $\mathrm{Na}_{2} \mathrm{SO}_{4}-\mathrm{Li}_{2} \mathrm{SO}_{4}$ Solution

\begin{tabular}{|c|r|r|c|}
\hline Glass & $\mathrm{T}\left({ }^{\circ} \mathrm{C}\right)$ & wt.\% $\mathrm{SO}_{3}$ in glass & $\begin{array}{c}\text { wt.\% } \mathrm{Li}_{2} \mathrm{SO}_{4} \text { in } \\
\text { equilibrium molten } \\
\text { salt phase }\end{array}$ \\
\hline WL-3 & 900 & 0.95 & 9.8 \\
& 1150 & 1.36 & 13.7 \\
& 1400 & 1.51 & 16.9 \\
\hline WL-4 & 900 & 0.95 & 9.3 \\
& 1150 & 1.37 & 13.1 \\
& 1400 & 1.56 & 16.4 \\
\hline WL-5 & 900 & 0.93 & 8.8 \\
& 1150 & 1.39 & 12.6 \\
& 1400 & 1.61 & 15.9 \\
\hline
\end{tabular}




\section{Table 15}

Base Composition of Simulated Low Level Nuclear Waste Glass Used in Studies of Crichton ef al 194 Cril

\begin{tabular}{|c|c|c|c|c|c|}
\hline Component & wt.\% & Component & wt. \% & Component & wt.\% \\
\hline $\mathrm{SiO}_{2}$ & 56.78 & $\mathrm{P}_{2} \mathrm{O}_{5}$ & 1.187 & $\mathrm{Fe}_{2} \mathrm{O}_{3}$ & 0.005 \\
$\mathrm{~B}_{2} \mathrm{O}_{3}$ & 5.00 & $\mathrm{~F}$ & 0.213 & $\mathrm{Cr}_{2} \mathrm{O}_{3}$ & 0.036 \\
$\mathrm{Na}_{2} \mathrm{O}$ & 20.00 & $\mathrm{Cl}$ & 0.092 & $\mathrm{Nd}_{2} \mathrm{O}_{3}$ & 0.012 \\
$\mathrm{Al}_{2} \mathrm{O}_{3}$ & 12.00 & $\mathrm{SO}_{3}$ & 0.321 & $\mathrm{Bi}_{2} \mathrm{O}_{3}$ & 0.014 \\
$\mathrm{CaO}$ & 4.00 & $\mathrm{~K}_{2} \mathrm{O}$ & 0.327 & $\mathrm{ZrO}_{2}$ & 0.005 \\
\hline
\end{tabular}


$\mathrm{SO}_{3}$ solubilities in glass in equilibrium with a molten sulfate phase have also been reported. Kordes et al [ 51 Kor] (quoted by [66 Holl) reports solubilities of $\mathrm{Na}_{2} \mathrm{SO}_{4}$ of 7 and $3 \mathrm{wt} . \%$ in $\mathrm{Na}_{2} \mathrm{O} \cdot 2 \mathrm{SiO}_{2}$ and $\mathrm{Na}_{2} \mathrm{O} \cdot 3 \mathrm{SiO}_{2}$ respectively at $1200^{\circ} \mathrm{C}$ in equilibrium with liquid $\mathrm{Na}_{2} \mathrm{SO}_{4}$. Calculations give 9.4 and 4.8 wt.\% respectively. Pearce and Beisler [65 Pea] indicate a composition containing $-3 \mathrm{wt}$. $\% \mathrm{Na}_{2} \mathrm{SO}_{4}$ at the glass/tridymite $/ \mathrm{Na}_{2} \mathrm{SO}_{4}$ (liq.) triple point at $1200^{\circ} \mathrm{C}$. Calculations give 2.6 wt. $\%$.

For the three alkali borosilicate glass compositions listed in Table 14, Sullivan 194 Sul] reports a solubility of $\mathrm{SO}_{3}$ (in equilibrium with a molten sulfate phase) of $1.3 \mathrm{wt} \% \mathrm{SO}_{3}$ for glass WL-3 and 1.1 and $1.2 \mathrm{wt} . \%$ for glasses WL-4 and WL-5 respectively, without specifying the temperature. Calculated solubilities in Table 14 are in very good agreement with the results of Sullivan. It can be seen that the calculated solubilities are quite insensitive to temperature (as expected for equilibrium between two liquid phases).

Finally, Crichton et al [94 Cri] report a "solubility" of $0.68 \mathrm{wt.} \% \mathrm{SO}_{3}$ in equilibrium with $\mathrm{Na}_{2} \mathrm{SO}_{4}$ (temperature unspecified) for an alkali borosilicate glass of the composition given in Table 15. Calculations (ignoring minor constituents) gave $2.4,2.2$ and $2.1 \mathrm{wt} \% \mathrm{SO}_{3}$ at $900^{\circ}$, $1150^{\circ}$ and $1400^{\circ} \mathrm{C}$ respectively. This agreement is not as good, but the description of the experimental method as given by Crichton et al is not at all clear.

\subsection{Phosphate solubilities}

For phosphate solubilities in glasses there are very few measurements, and these are often contradictory and of poor accuracy. The thermodynamic properties of the pure phosphates are also not well known.

Thermodynamic data for pure liquid $\mathrm{Mg}_{3}\left(P \mathrm{O}_{4}\right)_{2}$ were taken from the $F$ *A*C*T database. Data for solid $\mathrm{Ca}_{3}\left(\mathrm{PO}_{4}\right)_{2}$ and $\mathrm{Na}_{3}\left(\mathrm{PO}_{4}\right)$ were found, but no data for the liquids were found and so these were estimated from the solid data and from estimated Gibbs energies of fusion. For $\mathrm{Li}_{3}\left(\mathrm{PO}_{4}\right)$, only the enthalpy of formation at $298 \mathrm{~K}$ was found. Properties of the liquid were estimated by assuming that $\Delta S$ and $\Delta C p$ for formation of $\mathrm{Li}_{3}\left(\mathrm{PO}_{4}\right)$ from $\mathrm{Li}_{2} \mathrm{O}$ and $\mathrm{P}_{2} \mathrm{O}_{5}$ are the same as for the formation of $\mathrm{Na}_{3}\left(\mathrm{PO}_{4}\right)$ from $\mathrm{Na}_{2} \mathrm{O}$ and $\mathrm{P}_{2} \mathrm{O}_{5}$.

No adjustable model parameters were used.

Crichton et al [94 Cri] report a solubility limit of $3.0 \mathrm{wt} . \% \mathrm{P}_{2} \mathrm{O}_{5}$ in a glass of the composition given in Table 15 when in equilibrium with pure solid $\mathrm{Na}_{3} \mathrm{PO}_{4}$ and $\mathrm{Na}_{2} \mathrm{Ca}_{4}\left(\mathrm{PO}_{3}\right)_{2} \mathrm{SiO}_{4}$. They do not indicate the temperature for this result, and the description of their experiment is not clear. Calculations give $\mathrm{P}_{2} \mathrm{O}_{5}$ contents at $700^{\circ}, 900^{\circ}, 1100^{\circ}, 1300^{\circ}$ and $1500^{\circ} \mathrm{C}$ of $0.13,0.74,1.98,2.9$ and $3.8 \mathrm{wt} . \%$ respectively in equilibrium with solid $\mathrm{Na}_{3}\left(\mathrm{PO}_{4}\right)$ and $\mathrm{Ca}_{3}\left(\mathrm{PO}_{4}\right)_{2}$. 
Phosphate solubilities in $\mathrm{Na}_{2} \mathrm{O}-\mathrm{SiO}_{2}$ glasses in equilibrium with a gas phase are reported by Inoue and Suito $[85 \mathrm{Ino}]$. These are reported as "phosphate capacities", $\mathrm{C}_{\mathrm{PO}_{4}}=\left(w t . \% \mathrm{PO}_{4}\right) / \mathrm{P}_{2}^{1 / 2} \bullet \mathrm{P}_{\mathrm{O}_{2}}^{5 / 4}\left(\mathrm{p}=\right.$ atm). At $1250^{\circ} \mathrm{C}$ in solutions with $\mathrm{SiO}_{2} / \mathrm{Na}_{2} \mathrm{O}$ molar ratios of $3 / 2$ and $1 / 1$, values of $\log \left(C_{P O_{4}}\right)=26.4$ and 25.4 respectively are reported, whereas present calculations give 27.2 and 24.6 respectively as shown in Table 16. Similar data from other sources have been collected by Ban-ya and Hino [91 Ban]. Discrepancies of this order are found between sources.

The calculated temperature dependence of $\mathrm{C}_{\mathrm{PO}_{4}}$ over the range $1200^{\circ}$ to $1350^{\circ} \mathrm{C}$ agrees well with the reported data [91 Ban].

Inoue and Suito [85 Ino] report phosphate capacities for $\mathrm{Na}_{2} \mathrm{O}-\mathrm{SiO}_{2}$ glasses with additions of $\mathrm{Mg}$ and $\mathrm{Ca}$ silicates. These are shown in Table 16 where it can be seen that agreement with calculated values is satisfactory. 


\section{Table 16}

Calculated Phosphate Capacities $\mathrm{C}_{\mathrm{PO}_{4}}$ in Glasses at $1250^{\circ} \mathrm{C}$ Compared with Measurements of Inoue and Suito $185 \mathrm{Inol}$

$$
C_{\mathrm{PO}_{4}}=\left(w t . \% \mathrm{PO}_{4} / \mathrm{P}_{\mathrm{P}_{2}}^{1 / 2} \mathrm{P}_{\mathrm{O}_{2}}^{5 / 4} \text { ) where } \mathrm{P}=\right.\text { atm }
$$

\begin{tabular}{|c|c|c|c|c|}
\hline \multicolumn{3}{|c|}{ Composition (wt.\%) } & \multicolumn{2}{|c|}{$\log \left(C_{\mathrm{PO}_{4}}\right)$} \\
\hline $\begin{array}{l}3 \mathrm{Na}_{2} \mathrm{O} \\
.2 \mathrm{SiO}_{2} \\
\end{array}$ & $3 \mathrm{CaO} \cdot 2 \mathrm{SiO}_{2}$ & $3 \mathrm{MgO} .2 \mathrm{SiO}_{2}$ & expt. & calc. \\
\hline $\begin{array}{r}100 \\
80 \\
80 \\
\end{array}$ & $\begin{array}{r}0 \\
20 \\
0\end{array}$ & $\begin{array}{r}0 \\
0 \\
20\end{array}$ & \multirow[t]{2}{*}{$\begin{array}{l}26.4 \\
26.2 \\
25.7\end{array}$} & \multirow[t]{2}{*}{$\begin{array}{l}27.2 \\
26.4 \\
24.3\end{array}$} \\
\hline $\mathrm{Na}_{2} \mathrm{O} \cdot \mathrm{SiO}_{2}$ & $\mathrm{CaO} \cdot \mathrm{SiO}_{2}$ & $\mathrm{MgO} . \mathrm{SiO}_{2}$ & & \\
\hline $\begin{array}{r}100 \\
80 \\
80\end{array}$ & $\begin{array}{r}0 \\
20 \\
0\end{array}$ & $\begin{array}{r}0 \\
0 \\
20\end{array}$ & $\begin{array}{l}25.4 \\
24.85 \\
24.7\end{array}$ & $\begin{array}{l}24.6 \\
24.1 \\
23.5\end{array}$ \\
\hline
\end{tabular}




\subsection{Halide solubilities}

The only data which could be found on the solubilities of halides are those of Crichton et al [94 Cri]. As already mentioned above, the descriptions of the experiments are confusing, and in the case of sulfate solubilities Crichton et al do not agree well with Sulivan [94 Sul] who studied glasses of similar composition.

For the model calculations, all $G^{\circ}$ values of the pure halides were taken from the $F * A * C * T$ database.

For the case of chloride solubilty, no adjustable model parameters were required. At $900^{\circ} \mathrm{C}, 1100^{\circ} \mathrm{C}$ and $1300^{\circ} \mathrm{C}$, the $\mathrm{Cl}$ content of a glass of the composition given in Table 15 (ignoring minor components) was calculated as $0.77,0.78$ and $0.79 w t \%$ respectively when in equilibrium with liquid $\mathrm{NaCl}$. Crichton et al, without giving a temperature, report a solubility of 0.8 wt. $\%$ in excellent agreement with the calculations. The insensitivity of the solubility to change in temperature is as expected for equilibrium between two liquid phases. Crichton et al also report solubilities as a function of temperature over the range $800^{\circ} \mathrm{C}$ to $1300^{\circ} \mathrm{C}$, but it is not possible to decipher from their description exactly what they measured nor exactly what the results were. In any case, they observed the solubility to be insensitive to temperature changes, in agreement with the calculations.

For the case of iodide solubility, Crichton et al report solubilities of $0.5 \pm 0.3 w t . \%$ I (depending on the experimental method) when a slag of the composition of Table 1 is in equilibrium with liquid Nal. With no adjustable model parameters, a solubility of $-2.0 \%$ was calculated. An adjustable parameter of $R T I n \gamma_{\mathrm{Na}}=3500 \mathrm{cal} / \mathrm{mol}$ was thus introduced, resulting in calculated solubilities of $0.37,0.53$ and $0.69 \mathrm{wt} . \%$ at $700^{\circ}, 900^{\circ}$ and $1100^{\circ} \mathrm{C}$ respectively. Such a small positive parameter is reasonable in view of the large size of the iodide ion which would tend to decrease its solubility. Similar parameters $R T \ln y_{i}=3500$ $\mathrm{cal} / \mathrm{mol}$ were also included for $\mathrm{Ca}_{1 / 2} \mathrm{l}, \mathrm{Lil}$ and $\mathrm{Mg}_{1 / 2} \mathrm{l}$ in the glass.

For the case of fluoride solubility, the glass is in equilibrium with solid fluorides over the temperature range of the experiments of Crichton et al. Hence, the solubility will depend more strongly on temperature. These authors report a solubility of 2.8 wt.\% $F$ in a slag of the composition given in Table 15. This seems to be the value observed at $725^{\circ} \mathrm{C}$ (see their figure 2). With no adjustable parameters, a solubility of $0.6 \mathrm{wt} . \%$ was calculated at $725^{\circ} \mathrm{C}$ in equilibrium with solid NaF and solid $\mathrm{CaF}_{2 \cdot}$. Accordingly, an adjustable parameter of $R T I n Y_{\text {NuF }}=-3500 \mathrm{cal} / \mathrm{mol}$ was introduced, resulting in a calculated solubility of $2.65 \mathrm{wt} . \%$ at $725^{\circ} \mathrm{C}$. Such a small negative parameter is reasonable in view of the fact that fluorine can probably enter the glass not only as $\mathrm{F}^{-}$ions but also by bonding directly to Si, thereby increasing its solubility. Similar parameters $R T \ln \mathrm{Y}_{\mathrm{N}_{\mathrm{ff}}}=-3500 \mathrm{cal} / \mathrm{mol}$ were also included for $\mathrm{Ca}_{1 / 2} \mathrm{I}$, Lil and $\mathrm{Mg}_{1 / 2} \mathrm{l}$ in the glass. 
The calculations give a solubility of $8.7 \mathrm{~mol} \% \mathrm{~F}(2.65 \mathrm{wt} . \% \mathrm{~F})$ at $725^{\circ} \mathrm{C}$ and $10.1 \mathrm{~mol} \% \mathrm{~F}$ at $850^{\circ} \mathrm{C}$ in equilibrium with solid $\mathrm{NaF}$ and $\mathrm{CaF}_{2 .}$. Crichton et al, in their figure 2. show an "immiscibility boundary" of $8.9 \mathrm{~mol} \%$ at $725^{\circ} \mathrm{C}$ and $-13.5 \mathrm{~mol} \%$ at $850^{\circ} \mathrm{C}$. However, the interpretation of this figure, and how it was obtained, are unclear.

\section{$9.4 \quad \mathrm{H}_{2} \mathrm{O}$ Solubilities}

In basic glasses, water is presumed to dissolve principally as free $\mathrm{OH}^{\circ}$ ions. However, in acid glasses, the hydroxyl groups are most likely bonded to silicon (-Si-O-H). This is evidenced by the observation in several binary glass systems $\mathrm{SiO}_{2}-\mathrm{M}_{2} \mathrm{O}(\mathrm{M}=\mathrm{Li}, \mathrm{Na}, \mathrm{K}), \mathrm{SiO}_{2}^{-}$ $\mathrm{CaO}$, etc. that as the $\mathrm{SiO}_{2}$ content is increased the $\mathrm{H}_{2} \mathrm{O}$ solubility at first decreases, but then passes through a minimum to increase again at high $\mathrm{SiO}_{2}$ contents.

For the fraction which dissolves as free $\mathrm{OH}^{-}$ions, the same model was used as was used for sulfate, phosphate and halide solubilities. For the fraction which dissolves as bonded hydroxy ions, a species " $\mathrm{H}_{2} \mathrm{O}$ ", formally similar to $\mathrm{Na}_{2} \mathrm{O}$, was introduced into the quasichemical model (with a quasichemical "charge parameter" of 0.3444 ).

Solubilities of $\mathrm{H}_{2} \mathrm{O}$ in different binary glasses have been measured by several authors. These are generally in good agreement and have been compiled by Ban-ya and Hino [91 Ban]. Some representative results are shown in Table 17. The following model parameters were chosen so as to optimize these data: $\mathrm{G}^{\circ}$ of liquid $\mathrm{NaOH}$ and $\mathrm{KOH}$ were taken from the $F^{*} A$ *C*T database. $G^{\circ}$ of liquid $\mathrm{Ca}(\mathrm{OH})_{2}$ and $\mathrm{Mg}(\mathrm{OH})_{2}$ were set equal to the values of the corresponding solids from the $F * A * C * T$ database. The following adjustable parameters were then introduced: $R T I n \gamma_{i}=-5.4,-18.0,-13.0$ and $-13.0 \mathrm{kcal} / \mathrm{mol}$ for $\mathrm{i}=\mathrm{NaOH}, \mathrm{LiOH}, \mathrm{Ca}_{1 / 2} \mathrm{OH}$ and $\mathrm{Mg}_{1 / 2} \mathrm{OH}$ respectively. For pure $\mathrm{H}_{2} \mathrm{O}, \mathrm{G}^{\circ}$ was set equal to the value obtained by extrapolating $\mathrm{G}^{\circ}$ of liquid $\mathrm{H}_{2} \mathrm{O}$ to high temperature and adding $30.0 \mathrm{kcal} / \mathrm{mol}$. This is, of course, necessarily an adjustable parameter of the model, but this procedure was chosen so as to give a reasonable temperature dependence. Finally, the following optimized quasichemical parameters were introduced:

$$
\begin{aligned}
& \omega_{\mathrm{HO}_{1 / 2} \mathrm{SiO}}=-22.0 \mathrm{kcal} / \mathrm{mol} \\
& \omega_{H O_{1 / 2} \mathrm{AlO} / 2}=-22.0 \mathrm{kcal} / \mathrm{mol}
\end{aligned}
$$

Calculated $\mathrm{H}_{2} \mathrm{O}$ solubilities are compared with reported values in Table 17 for binary glasses and with values for a ternary glass in Table 18. 


\section{Table 17} Measurements

Calculated $\mathrm{H}_{2} \mathrm{O}$ Capacities, $\mathrm{C}_{\mathrm{H}_{2}}$ or of Binary Glasses Compared to

$$
\mathrm{C}_{\mathrm{H}_{2} \mathrm{O}}=\text { (wt. } \% \mathrm{H}_{2} \mathrm{O} / \mathrm{P}_{\mathrm{H}_{2} \mathrm{O}}^{1 / 2} \text { ) where } \mathrm{P}=\text { atm }
$$

\begin{tabular}{|c|c|c|c|c|c|}
\hline \multirow{2}{*}{ System } & \multirow{2}{*}{$\begin{array}{l}\text { Mole Fraction } \\
\mathbf{X}_{\mathrm{SiO}_{2}} \text { or } \mathrm{X}_{\mathrm{N}_{2} \mathrm{O}_{3}}\end{array}$} & \multirow{2}{*}{$T^{\circ} \mathrm{C}$} & \multicolumn{2}{|c|}{$\log C_{\mathrm{H}_{2} \mathrm{O}}$} & \multirow[b]{2}{*}{ ref. } \\
\hline & & & $\begin{array}{c}\text { expt. } \\
\text { (smoothed) }\end{array}$ & calc. & \\
\hline $\mathrm{Na}_{2} \mathrm{O}-\mathrm{SiO}_{2}$ & $\begin{array}{l}0.85 \\
0.80 \\
0.75 \\
0.70 \\
0.50\end{array}$ & 1317 & $\begin{array}{l}-0.96 \\
-1.10 \\
-1.12 \\
-1.08 \\
-0.69 \\
\end{array}$ & $\begin{array}{l}-1.10 \\
-1.15 \\
-1.24 \\
-1.36 \\
-0.69 \\
\end{array}$ & [58 Kur] \\
\hline \multirow[t]{2}{*}{$\mathrm{Li}_{2} \mathrm{O}-\mathrm{SiO}_{2}$} & $\begin{array}{l}0.80 \\
0.69 \\
0.50\end{array}$ & 1300 & $\begin{array}{c}-0.90 \\
-0.97 \\
(-0.65)\end{array}$ & $\begin{array}{l}-1.19 \\
-1.13 \\
-0.59\end{array}$ & [58 Kur] \\
\hline & 0.667 & $\begin{array}{r}1394 \\
1156 \\
977 \\
\end{array}$ & $\begin{array}{r}-0.96 \\
-1.14 \\
-1.30 \\
\end{array}$ & $\begin{array}{l}-1.05 \\
-1.15 \\
-1.23 \\
\end{array}$ & [57 Rus] \\
\hline $\mathrm{CaO}-\mathrm{SiO}_{2}$ & $\begin{array}{l}0.6 \\
0.5 \\
0.4 \\
\end{array}$ & 1600 & $\begin{array}{l}-1.19 \\
-1.21 \\
-1.05 \\
\end{array}$ & $\begin{array}{r}-1.15 \\
-1.20 \\
-1.02 \\
\end{array}$ & [69 lgu] \\
\hline $\mathrm{CaO}-\mathrm{Al}_{2} \mathrm{O}_{3}$ & $\begin{array}{l}0.5 \\
0.4 \\
0.3\end{array}$ & 1650 & $\begin{array}{l}-1.00 \\
-0.84 \\
-0.55\end{array}$ & $\begin{array}{l}-1.37 \\
-0.80 \\
-0.42\end{array}$ & [90 Wat] \\
\hline
\end{tabular}




\section{Table 18}

Calculated $\mathrm{H}_{2} \mathrm{O}$ Capacities, $\mathrm{C}_{\mathrm{H}_{2}}$ o of $\mathrm{SiO}_{2}-\mathrm{MgO}-\mathrm{CaO}$ Glasses at $1450^{\circ} \mathrm{C}$ with $\mathrm{X}_{\mathrm{SiO}_{2}}=0.50$ Compared to Measurements of Ban-ya et al [86 Ban]

$$
\mathrm{C}_{\mathrm{H}_{2} \mathrm{O}}=\text { (wt. } \% \mathrm{H}_{2} \mathrm{O} / \mathrm{P}_{\mathrm{H}_{2} \mathrm{O}}^{1 / 2} \text { ) where } \mathrm{P}=\text { atm }
$$

\begin{tabular}{|r|r|r|}
\hline \multirow{2}{*}{$\begin{array}{c}\text { Molar Ratio } \\
\text { MgO/lMgO + CaO) }\end{array}$} & \multicolumn{2}{|c|}{$\log \mathrm{C}_{\mathrm{H}_{2} \mathrm{O}}$} \\
\cline { 2 - 4 } & expt. & calc. \\
\hline 0.25 & $\sim(-1.13)$ & -1.16 \\
0.375 & -1.12 & -1.14 \\
0.50 & -1.10 & -1.12 \\
0.625 & -1.08 & -1.10 \\
0.75 & $-(-1.05)$ & -1.07 \\
\hline
\end{tabular}




\section{MOLTEN SALT DATABASES}

Databases have been developed for molten salt solutions which can exist in equilibrium with glasses. A sublattice model was used which has been described previously [ 88 Pel]. Databases were developed by optimization of thermodynamic and phase diagram data for all binary and several common-ion ternary systems. Many of these optimizations have been described in publications [87San, 88Pel, 90Des1,90Des2,90Des3, $91 \mathrm{San}$ ] and several others have been more recently performed. In general, the model for molten salts is good, the optimizations reproduce the data very well, and there are many data available. Hence, the thermodynamic properties of the molten salt solutions are expected to be well modeled.

Two solution databases should be used in the calculations. The first, developed as a private database for Battelle, is the $\mathrm{Li}, \mathrm{Na}, \mathrm{Mg}, \mathrm{Ca} / \mathrm{F}, \mathrm{SO}_{4}$ solution. That is, this database models a molten salt solution containing the cations $\mathrm{Li}^{+}, \mathrm{Na}^{+}, \mathrm{Mg}^{2+}$, and $\mathrm{Ca}^{2+}$ and the anions F- and $\mathrm{SO}_{4}^{2-}$. The other solution is the $\mathrm{F}^{*} \mathrm{~A}$ "C*T public solution database /SOLN-SALT/ which models the $\mathrm{Li}, \mathrm{Na} / \mathrm{F}, \mathrm{Cl}, \mathrm{I}, \mathrm{SO}_{4}$ molten phase. 
11 REFERENCES

[30 Bow] N.L. Boiven, J. F. Schairer and H.W.V. Willems, Am. J. Sci., 20, 405-455 (1930).

[51 Kor] E. Kordes, B. Zöfelt and H. Pröger, Z. Anorg. Allg. Chem., 264, 255-271 (1951).

[51 Mor] G.W. Morey, J. Soc. Glass Tech., 3ㅗ., 270-284 (1951).

[54 Fin] C.J.B. Fincham and F.D. Richardson, Proc. Roy. Soc. London, A223, 40-62 (1954).

[57 Rus] L.E. Russell, J. Soc. Glass Tech., 41, 304T (1957).

[58 Kur] C. R. Kurkjian and L.E. Russell, J. Soc. Glass Tech., 42, $130 T$ (1958).

[60 Osb] E. F. Osborn and A. Muan, "Phase Equilibrium Diagrams of Oxide Systems", Am. Ceram. Soc. (1960).

[65 Pea] M.L. Pearce and J.F. Beisler, J. Am. Ceram. Soc., 48, 40-42 (1965).

[66 Holl S. Holmquist, J. Am. Ceram. Soc., 49, 467-473 (1966).

169 Igu] Y. Iguchi, S. Ban-ya and T. Fuwa, Trans. ISIJ, 9, 189 (1969).

173 Nagl S. Nagashima and T. Katsura, Bull. Chem. Soc. Jap., 46, 3099-3103 (1973).

[73 Papl K. Papadopoulos, Phys. Chem. Glasses, 14, 60-65 (1973).

[85 Ino] R. Inoue and H. Suito, Trans. ISIJ, 25, 118 (1985).

[86 Ban] S. Ban-ya, Y. Iguchi and S. Yamamoto, Tetsu-to-Hagané, 72, 2210 (1986).

187 San]. J. Sangster and A.D. Pelton, J. Phys. Chem. Ref. Data, 16, 509-561 (1987).

[87 Sch] H.D.Schreiber, S. Kozak, P.G. Leonhard and K.K. McManus, Glastech. Ber., 60, 389-398 (1967).

[88 Pell A.D. Pelton, Calphad J., 12, 127-142 (1988).

[90 Des1] Y. Dessureault, J. Sangster and A.D. Pelton, J. Electrochem, Soc., 137, 29412950 (1990). 
[90 Des2] Y. Dessureault, J. Sangster, and A.D. Pelton, J. Phys. Chem. Ref. Data, 119 , $1149-1178$ (1990).

190 Des3] Y. Dessureault, J. Sangster and A.D. Pelton, J. Chim. Phys., 87, 407-453 (1990)..

[90 Holl Holland and Powell, J. Metamorph. Geol., ․, 89-124 (1990).

190 Wat] M. Watanabe, Y. Iguchi and S. Ban-ya, Tetsu-to-Hagané, 76, 1672 (1990).

[91 Banl S. Ban-ya and M. Hino, "Chemical Properties of Molten Slags", Iron and Steel Inst. Japan, Tokyo(1991).

[91 San] J. Sangster and A.D. Pelton, J. Phase Equilib., 12, $511-537$ (1991).

192 Pell A.D. Pelton, P. Wu and G. Eriksson, "Development of Models and Software for Liquidus Temperatures of Glasses of HWVP Products, "Report to Battelle, (November, 1992).

[93 Pel] A.D. Pelton, G. Eriksson and J. A. Romero-Serrano, Metall. Trans., 24B, 817-825 (1993).

194 Cri] S.N. Crichton, T. J. Barbieri and M. Tomozawa, "Solubility Limits for Troublesome Components in a Simulated Low Level Nuclear Waste Glass", manuscript communicated by P. Hrma (1994).

[94 Sul] G.K. Sullivan, "Sulfate Segregation in Vitrification of Simulated Hanford Nuclear Waste Glass", manuscript communicated by P. Hrma (1994). 
$\because \dot{0}$ 